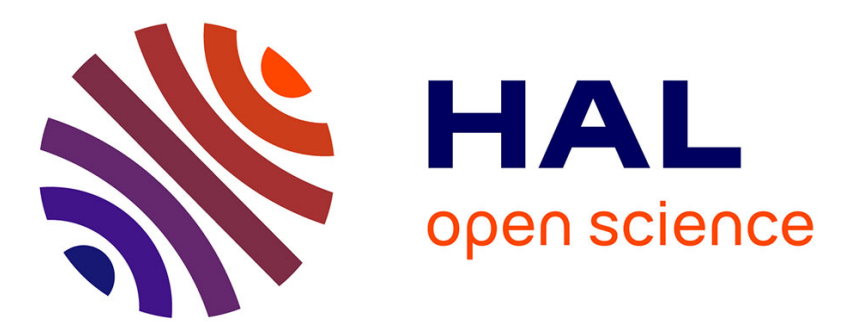

\title{
Numerical observers with vanishing viscosity for the 1d wave equation
}

\author{
Galina Garcia, Takéo Takahashi
}

\section{To cite this version:}

Galina Garcia, Takéo Takahashi. Numerical observers with vanishing viscosity for the $1 \mathrm{~d}$ wave equation. Advances in Computational Mathematics, 2014, 40 (4), pp.711-745. 10.1007/s10444-013-93205. hal-00914924

\author{
HAL Id: hal-00914924 \\ https://hal.science/hal-00914924
}

Submitted on 10 Dec 2013

HAL is a multi-disciplinary open access archive for the deposit and dissemination of scientific research documents, whether they are published or not. The documents may come from teaching and research institutions in France or abroad, or from public or private research centers.
L'archive ouverte pluridisciplinaire HAL, est destinée au dépôt et à la diffusion de documents scientifiques de niveau recherche, publiés ou non, émanant des établissements d'enseignement et de recherche français ou étrangers, des laboratoires publics ou privés. 


\title{
Numerical observers with vanishing viscosity for the $1 \mathrm{~d}$ wave equation
}

\author{
Galina García*, Takéo Takahashi ${ }^{\dagger \S}$
}

July 7,2013

\begin{abstract}
We consider a numerical scheme associated with the iterative method developed in [34] to recover initial conditions of conservative systems. In this method, the initial conditions are reconstructed by using observers. Here we use a finite-difference discretization in space of these observers and our aim is to prove estimates of the errors with respect to the mesh size and to the number of steps in the iterative method. This is done in the particular example of the $1 \mathrm{~d}$ wave equation. In order to avoid restrictions of the number of steps with respect to the mesh size, we add a numerical viscosity in the numerical observers. A generalization for other equations is also given.
\end{abstract}

\section{Introduction and main results}

The aim of this article is to consider and analyze an iterative method based on observers to reconstruct initial conditions of some evolution equations. More precisely, we focus on a method developed in [34] to recover the initial conditions for reversible infinite-dimensional systems. Our aim is to analyze this method when we discretize the evolution equations. In order to do this, we recall the method considered in [34] for a 1d wave equation and we then discretize this equation by using the finite difference method on a uniform mesh. Nevertheless, our approach can be extended to other systems and to other numerical discretization methods (see Section 7).

Let us consider the $1 \mathrm{~d}$ wave equation:

$$
\begin{cases}\partial_{t t} v-\partial_{x x} v=0 & \text { in }(0, \tau) \times(0,1) \\ v(t, 0)=0 & \text { for } t \in(0, \tau), \\ \partial_{x} v(t, 1)=0 & \text { for } t \in(0, \tau) \\ v(0, x)=v^{0}(x), \quad \partial_{t} v(0, x)=v^{1}(x) & \text { for } x \in(0,1)\end{cases}
$$

It is well-known that system (1) is well-posed for

$$
\left(v^{0}, v^{1}\right) \in H_{L}^{1}(0,1) \times L^{2}(0,1),
$$

where

$$
H_{L}^{1}(0,1):=\left\{v \in H^{1}(0,1) ; v(0)=0\right\}
$$

(see, for instance, [5] for the definitions of the Lebesgue and the Sobolev spaces). In particular, for initial conditions satisfying (2), there exists a unique solution $v$ of system (1) verifying

$$
v \in C\left([0, \tau] ; H_{L}^{1}(0,1)\right) \cap C^{1}\left([0, \tau] ; L^{2}(0,1)\right) .
$$

\footnotetext{
* Departamento de Matemática y Ciencia de la Computación, Universidad de Santiago, Casilla 307, Correo 2, Santiago, Chile (galina.garcia@usach.cl)

${ }^{\dagger}$ Inria, Villers-lès-Nancy, F-54600, France (takeo.takahashi@inria.fr),

${ }^{\ddagger}$ Université de Lorraine, IECN, UMR 7502, Vandoeuvre-lès-Nancy, F-54506, France,

${ }^{\S}$ CNRS, IECN, UMR 7502, Vandoeuvre-lès-Nancy, F-54506, France,
} 
Moreover, if we define the observation $y$ by

$$
y(t):=\partial_{t} v(t, 1) \quad \text { for } t \in(0, \tau)
$$

then, from the admissibility of the observation operator (see, for instance, [37]), we have

$$
y \in L^{2}(0, \tau)
$$

The corresponding inverse problem consists in recovering the initial data $\left(v^{0}, v^{1}\right)$ of $(1)$ by using the observation $y$ defined by (3), where $\tau$ is a positive time to be determined.

In order to solve this inverse problem, Ramdani, Tucsnak and Weiss propose in [34] an iterative algorithm. They define the two following forward and backward operators $F_{y}, B_{y}$ :

$$
F_{y}: H_{L}^{1}(0,1) \times L^{2}(0,1) \rightarrow H_{L}^{1}(0,1) \times L^{2}(0,1), \quad\left(q^{0}, q^{1}\right) \mapsto\left(q(\tau), \partial_{t} q(\tau)\right),
$$

where

$$
\begin{cases}\partial_{t t} q-\partial_{x x} q=0 & \text { in }(0, \tau) \times(0,1), \\ q(t, 0)=0 & \text { for } t \in(0, \tau), \\ \left(\partial_{x} q+\partial_{t} q\right)(t, 1)=y(t) & \text { for } t \in(0, \tau) \\ q(0, x)=q^{0}(x), \quad \partial_{t} q(0, x)=q^{1}(x) & \text { for } x \in(0,1),\end{cases}
$$

and

$$
B_{y}: H_{L}^{1}(0,1) \times L^{2}(0,1) \rightarrow H_{L}^{1}(0,1) \times L^{2}(0,1), \quad\left(q_{b}^{0}, q_{b}^{1}\right) \mapsto\left(q_{b}(0), \partial_{t} q_{b}(0)\right),
$$

where

$$
\begin{cases}\partial_{t t} q_{b}-\partial_{x x} q_{b}=0 & \text { in }(0, \tau) \times(0,1) \\ q_{b}(t, 0)=0 & \text { for } t \in(0, \tau) \\ \left(\partial_{x} q_{b}-\partial_{t} q_{b}\right)(t, 1)=-y(t) & \text { for } t \in(0, \tau) \\ q_{b}(\tau, x)=q_{b}^{0}(x), \quad \partial_{t} q_{b}(\tau, x)=q_{b}^{1}(x) & \text { for } x \in(0,1)\end{cases}
$$

Then, they consider the sequences

$$
\left(a^{(n)}, b^{(n)}\right)=\left(B_{y} \circ F_{y}\right)\left(\left(a^{(n-1)}, b^{(n-1)}\right)\right), \quad n \in \mathbb{N}^{*},
$$

where $\left(a^{(0)}, b^{(0)}\right)$ is an initial guess of $\left(v^{0}, v^{1}\right)$.

The convergence analysis developed in [34] shows that for $\tau$ large enough, the sequence $\left(a^{(n)}, b^{(n)}\right)$ converges towards $\left(v^{0}, v^{1}\right)$. More precisely, we have the following result:

Proposition 1.1. For $\tau>2$, there exists $\alpha \in(0,1)$ such that for all $n \in \mathbb{N}$,

$$
\left\|a^{(n)}-v^{0}\right\|_{H_{L}^{1}(0,1)}^{2}+\left\|b^{(n)}-v^{1}\right\|_{L^{2}(0,1)}^{2} \leqslant \alpha^{2 n}\left(\left\|a^{(0)}-v^{0}\right\|_{H_{L}^{1}(0,1)}^{2}+\left\|b^{(0)}-v^{1}\right\|_{L^{2}(0,1)}^{2}\right) .
$$

Although the proof of the above proposition is a consequence of the general results of [34], we give it in Section 2 since it is quite short in our case and contains hints for the proof of the main result.

Let us note that the condition $\tau>2$ is related to the exact observability of (1) with the observation (3). Indeed, (1), (3) is exactly observable if and only if $\tau>2$. For the wave equation in dimension $N>1$, the time $\tau$ is related to "Geometric Control Condition" (see [3]). We recall that exact observability is related to exact controllability and to stabilization property (see [21], [37]).

In this paper, we focus on the discretized counterpart of the above result. Let $N \in \mathbb{N}$ and

$$
h=\frac{1}{N+1} .
$$

We consider the uniform subdivision of $(0,1): x_{j}=j h, j=0, \ldots,(N+1)$. Finally, we consider a positive constant $\nu$ that is used in the numerical scheme to add a numerical viscosity. 
The numerical adaptation of the method of [34] is given below. First, we consider spatial approximations of the forward and backward operators:

$$
F_{y, h}: \mathbb{R}^{N+2} \times \mathbb{R}^{N+2} \rightarrow \mathbb{R}^{N+2} \times \mathbb{R}^{N+2}, \quad\left(q_{h}^{0}, q_{h}^{1}\right) \mapsto\left(q_{h}(\tau), q_{h}^{\prime}(\tau)\right),
$$

where $q_{h}=\left(q_{j}\right)_{0 \leqslant j \leqslant N+1}$ verifies

$$
\begin{cases}q_{j}^{\prime \prime}-\frac{q_{j+1}-2 q_{j}+q_{j-1}}{h^{2}}-\nu\left(q_{j+1}^{\prime}-2 q_{j}^{\prime}+q_{j-1}^{\prime}\right)=0 & \text { in }(0, \tau), 1 \leqslant j \leqslant N, \\ q_{0}=0 & \text { in }(0, \tau), \\ \frac{q_{N+1}-q_{N}}{h}+q_{N+1}^{\prime}=y & \text { in }(0, \tau), \\ q_{j}(0)=q_{j}^{0}, \quad q_{j}^{\prime}(0)=q_{j}^{1} & \text { for } j=0, \cdots, N+1,\end{cases}
$$

and

$$
B_{y, h}: \mathbb{R}^{N+2} \times \mathbb{R}^{N+2} \rightarrow \mathbb{R}^{N+2} \times \mathbb{R}^{N+2}, \quad\left(q_{b, h}^{0}, q_{b, h}^{1}\right) \mapsto\left(q_{b, h}(0), q_{b, h}^{\prime}(0)\right),
$$

where $q_{b, h}=\left(q_{b, j}\right)_{0 \leqslant j \leqslant N+1}$ verifies

$$
\begin{cases}q_{b, j}^{\prime \prime}-\frac{q_{b, j+1}-2 q_{b, j}+q_{b, j-1}}{h^{2}}+\nu\left(q_{b, j+1}^{\prime}-2 q_{b, j}^{\prime}+q_{b, j-1}^{\prime}\right)=0 & \text { in }(0, \tau), 1 \leqslant j \leqslant N, \\ q_{b, 0}=0 & \text { in }(0, \tau), \\ \frac{q_{b, N+1}-q_{b, N}}{h}-q_{b, N+1}^{\prime}=-y & \text { in }(0, \tau), \\ q_{b, j}(\tau)=q_{b, j}^{0}, \quad q_{b, j}^{\prime}(\tau)=q_{b, j}^{1} & \text { for } j=0, \cdots, N+1 .\end{cases}
$$

Recall that $y(t)=\partial_{t} v(t, 1)$ is the observation of the system (1) defined by (3).

The terms $-\nu\left(q_{j+1}^{\prime}-2 q_{j}^{\prime}+q_{j-1}^{\prime}\right)$ in (12) and $+\nu\left(q_{b, j+1}^{\prime}-2 q_{b, j}^{\prime}+q_{b, j-1}^{\prime}\right)$ in (14) are numerical viscosities that play crucial role in our analysis. The idea of adding numerical viscosities in the numerical schemes for the wave equation was already considered by [35], [33], [31], [29], etc. They use these viscosities to obtain uniform observability or stabilization properties for the discretization of the wave equation. Without these viscosities, these properties may fail because of some spurious modes which are not well approximated ([2], [17], [18], etc.). Here, to reconstruct the initial conditions, a key point in the method is to have a uniform decay of the energy in time $\tau$ and this property is strongly related to the stabilization, which explain that we also add some numerical viscosity in the above systems. The change of sign is simply coming from the method proposed in [34], where both the forward and the backward steps need these uniform decay of the energy.

Using these operators $F_{y, h}$ and $B_{y, h}$, we can recover the approximations of $v^{0}$ and $v^{1}$ by using the following sequences:

$$
\left(a_{h}^{(n)}, b_{h}^{(n)}\right)=\left(B_{y, h} \circ F_{y, h}\right)\left(\left(a_{h}^{(n-1)}, b_{h}^{(n-1)}\right)\right), \quad n \in \mathbb{N}^{*}
$$

where $\left(a_{h}^{(0)}, b_{h}^{(0)}\right)$ is an initial guess of $\left(v_{h}^{0}, v_{h}^{1}\right)$.

Our main result is

Theorem 1.2. Assume that $v^{0} \in H^{2}(0,1) \cap H_{L}^{1}(0,1)$ and that $v^{1} \in H_{L}^{1}(0,1)$. For $\tau>2$ and for $\nu>0$, there exists $\gamma=\gamma(\tau, \nu) \in(0,1)$ such that for all $N \in \mathbb{N}$ (and $h$ given by (10)) and for all $n \in \mathbb{N}$,

$$
\begin{aligned}
& \frac{h}{2} \sum_{j=0}^{N}\left[\left(b_{j}^{(n)}-v_{j}^{1}\right)^{2}+\left(\frac{a_{j+1}^{(n)}-a_{j}^{(n)}}{h}-\frac{v_{j+1}^{0}-v_{j}^{0}}{h}\right)^{2}\right]+\frac{\nu h^{2}}{2}\left(b_{N+1}^{(n)}-v_{N+1}^{1}\right)^{2} \\
\leqslant & \frac{1}{1-\gamma^{2}} \varepsilon_{h}+\gamma^{2 n}\left(\frac{h}{2} \sum_{j=0}^{N}\left[\left(b_{j}^{(0)}-v_{j}^{1}\right)^{2}+\left(\frac{a_{j+1}^{(0)}-a_{j}^{(0)}}{h}-\frac{v_{j+1}^{0}-v_{j}^{0}}{h}\right)^{2}\right]+\frac{\nu h^{2}}{2}\left(b_{N+1}^{(0)}-v_{N+1}^{1}\right)^{2}\right),
\end{aligned}
$$


where $v_{j}^{0}=v^{0}(j h), v_{j}^{1}=v^{1}(j h)$ for $0 \leqslant j \leqslant N+1$ and where

$$
\varepsilon_{h}=\varepsilon_{h}\left(\left\|v^{0}\right\|_{H^{2}(0,1) \cap H_{L}^{1}(0,1)},\left\|v^{1}\right\|_{H_{L}^{1}(0,1)}\right)
$$

is independent of $n$ and goes to 0 as $h \rightarrow 0$.

Remark 1.3. In the above result, let us note that in (15)

$$
\frac{h}{2} \sum_{j=0}^{N}\left[\left(b_{j}^{(n)}-v_{j}^{1}\right)^{2}+\left(\frac{a_{j+1}^{(n)}-a_{j}^{(n)}}{h}-\frac{v_{j+1}^{0}-v_{j}^{0}}{h}\right)^{2}\right]
$$

is a discrete counterpart of

$$
\left\|b^{(n)}-v^{1}\right\|_{L^{2}(0,1)}^{2}+\left\|a^{(n)}-v^{0}\right\|_{H_{L}^{1}(0,1)}^{2}
$$

in (9). The term $\frac{1}{1-\gamma^{2}} \varepsilon_{h}$ is independent of the number of iterations and can only be reduced by decreasing $h$, whereas the other term in the right-hand side of (15) goes to 0 if the number of iterations goes to infinity.

Remark 1.4. It is important to notice that the numerical viscosity we use in our numerical scheme allows to obtain in the above result a time $\tau$ and a decay $\gamma$ independent of $h$.

Remark 1.5. In Theorem 1.2, we need the following regularity on the initial conditions: $v^{0} \in$ $H^{2}(0,1) \cap H_{L}^{1}(0,1)$ and $v^{1} \in H_{L}^{1}(0,1)$. In particular, we need more regularity than in Proposition 1.1. This assumption allows us to deal with the numerical error for the observation (at the boundary), and it is not clear that it is possible to obtain (15) for $v^{0} \in H_{L}^{1}(0,1)$ and $v^{1} \in L^{2}(0,1)$. For locally distributed observation, we would obtain the result for $v^{0} \in H_{0}^{1}(0,1)$ and $v^{1} \in L^{2}(0,1)$.

Let us make some comments on the main result. We consider here a finite difference approximations of the iterative algorithm proposed by [34]. This algorithm use back and forth in time observers to reconstruct the initial conditions. The use of observers is classical in finite dimensional systems (see, for instance, [20] ) and has been tackled in infinite dimensional systems ([1], [4], [6], [7], [9], [30], [10], [19], [22], [25]). The algorithm using back and forth in time observers for finite dimensional systems was considered in [15].

The method we use here could be adapted to other systems (Schrödinger equation, plate equation, Maxwell system, etc.) Instead of adding a vanishing viscosity, we could use one of the several methods which have been developed in the literature to handle the high frequency spurious modes: Tychonoff regularization [11], mixed finite elements [2], filtering of high frequencies [18], [24], etc. (see the review paper [38] for more details and extensive references). Let us note that in [8], the authors, prove that for regular solutions, the numerical viscosity is not needed. We present in Section 7 some possible generalizations of the studied done for the $1 \mathrm{~d}$ wave equation.

It is important to notice that our work is related to another approach of this problem developed in [16]. In that case, the authors don't add any vanishing viscosity and analyze the corresponding convergence of the numerical observers towards the initial conditions. The main differences with our work are that in their cases, the initial data are more regular and the observability operator is bounded with an extra hypothesis of regularity. Indeed their proofs, based on finite element method, consists in using these regularity assumptions to derive estimates errors and to follow the result obtain in the continuous system. In particular, it allows them to treat with the same approach a large class of systems and also to obtain similar results for the full-discretized case.

Here, we work directly on the discrete systems, and thus we don't need such assumptions on the regularity of the initial conditions. In fact, we could adapt our work to the case of internal 
observation and in that case, we could work with initial condition only in $H_{0}^{1}(0,1) \times L^{2}(0,1)$ instead of $\left[H^{2}(0,1) \cap H_{0}^{1}(0,1)\right] \times H_{0}^{1}(0,1)$. Another difference with the result of [16] is that in their case, they need to choose a particular number of steps in the iterative method with respect to the mesh size. Here, since we add this numerical viscosity, such a relation is not needed.

The paper is organized as follows: in Section 2, we present a short proof of Proposition 1.1. An important ingredient to obtain this result is the study of a damped wave equation. The discretization of this damped wave equation is analyzed in Section 3. Then Section 4 is devoted to some properties of the numerical schemes associated to (1) if we add a numerical viscosity. We study in particular the convergence results and an admissibility property. This allows to prove Theorem 1.2 in Section 5. Section 6 gives some numerical examples of this method. Finally, Section 7 presents some possible extensions of the result obtained for the $1 \mathrm{~d}$ wave equation to more general systems.

\section{Analysis in the theoretical framework}

This section is devoted to the proof of Proposition 1.1. This analysis is already done in [34], but here we precise some aspects which help to understand the numerical analysis of the next section. Let us first consider the following damped wave equation.

$$
\begin{cases}\partial_{t t} w-\partial_{x x} w=0 & \text { in }(0, \infty) \times(0,1) \\ w(t, 0)=0 & \text { for } t \in(0, \infty) \\ \left(\partial_{x} w+\partial_{t} w\right)(t, 1)=0 & \text { for } t \in(0, \infty) \\ w(0, x)=w^{0}(x), \quad \partial_{t} w(0, x)=w^{1}(x) & \text { for } x \in(0,1)\end{cases}
$$

Then we have the following classical result:

Lemma 2.1. Assume $\tau>2$. Then there exists $\alpha \in(0,1)$ such that for all $\left(w^{0}, w^{1}\right) \in H_{L}^{1}(0,1) \times$ $L^{2}(0,1)$,

$$
\|w(\tau)\|_{H_{L}^{1}(0,1)}^{2}+\left\|\partial_{t} w(\tau)\right\|_{L^{2}(0,1)}^{2} \leqslant \alpha\left(\left\|w^{0}\right\|_{H_{L}^{1}(0,1)}^{2}+\left\|w^{1}\right\|_{L^{2}(0,1)}^{2}\right) .
$$

Remark 2.2. The proof we give for this lemma is classical and is based on the multiplier method (see, for instance, [21]). It is used to obtain stabilizability properties. We recall the proof here since we use a similar method in the numerical case. Let us also notice that this method can be used to prove the same result for the wave equation in several dimensions (see, for instance, [37]). For interior domain measurements, the same result can be obtained again by using a multiplier method but a compactness argument is needed (see [23] for the continuous case, [35] for the discretized case).

Proof. We first multiply the first equation of (16) by $\partial_{t} w$ and integrate over $(0,1)$ :

$$
\frac{d}{d t} \frac{1}{2} \int_{0}^{1}\left[\left(\partial_{t} w\right)^{2}+\left(\partial_{x} w\right)^{2}\right] d x-\partial_{x} w(1) \partial_{t} w(1)=0
$$

using the third equation in (17) we obtain

$$
\frac{d}{d t} \frac{1}{2} \int_{0}^{1}\left[\left(\partial_{t} w\right)^{2}+\left(\partial_{x} w\right)^{2}\right] d x+\left(\partial_{t} w\right)^{2}(1)=0
$$

which implies in particular that the energy

$$
E=\frac{1}{2} \int_{0}^{1}\left[\left(\partial_{t} w\right)^{2}+\left(\partial_{x} w\right)^{2}\right] d x
$$

is a nonincreasing function of time. 
Then we multiply the first equation of $(16)$ by $x \partial_{x} w$, integrate over $(0,1)$ and after some computation, we deduce

$$
\frac{d}{d t} \int_{0}^{1} \partial_{t} w x \partial_{x} w d x-\int_{0}^{1} x \partial_{x} \frac{\left(\partial_{t} w\right)^{2}}{2} d x-\int_{0}^{1} x \partial_{x} \frac{\left(\partial_{x} w\right)^{2}}{2} d x=0
$$

The above equation implies

$$
\frac{d}{d t} \int_{0}^{1}\left(\partial_{t} w\right) x\left(\partial_{x} w\right) d x-\left(\partial_{t} w\right)^{2}(1)+E(t)=0 .
$$

Let us set

$$
V_{\varepsilon}(t):=E(t)+\varepsilon \int_{0}^{1} \partial_{t} w(t) x \partial_{x} w(t) d x
$$

We deduce from (18) and (20) that

$$
V_{\varepsilon}^{\prime}(t)=(\varepsilon-1)\left(\partial_{t} w\right)^{2}(1)-\varepsilon E(t) .
$$

On the other hand, we have that

$$
\left|\int_{0}^{1} \partial_{t} w x \partial_{x} w d x\right| \leqslant E
$$

which implies that

$$
(1-\varepsilon) E \leqslant V_{\varepsilon} \leqslant(1+\varepsilon) E .
$$

Combining the above equation and (22) yields that for $\varepsilon \in(0,1)$,

$$
V_{\varepsilon}^{\prime}(t) \leqslant-\frac{\varepsilon}{1+\varepsilon} V_{\varepsilon}(t) \text { for all } t \geqslant 0 .
$$

We deduce from the above equation and (23) that

$$
E(t) \leqslant \frac{1+\varepsilon}{1-\varepsilon} \exp \left(-\frac{\varepsilon}{1+\varepsilon} t\right) E(0) .
$$

Let us consider the function

$$
f(\varepsilon):=\frac{1+\varepsilon}{\varepsilon} \ln \left(\frac{1+\varepsilon}{1-\varepsilon}\right) .
$$

For $\tau>f(\varepsilon)$, we have

$$
\alpha:=\frac{1+\varepsilon}{1-\varepsilon} \exp \left(-\frac{\varepsilon}{1+\varepsilon} \tau\right)<1,
$$

which implies (17). Since $\lim _{\varepsilon \rightarrow 0^{+}} f(\varepsilon)=2$, we conclude the proof of the lemma.

Using the above result, we deduce the proof of Proposition 1.1:

Proof of Proposition 1.1. Let us set $w=v-q$ where $v$ and $q$ are respectively the solutions of (1) and (5). Then it can be easily checked that $w$ is the solution of (16) with the initial conditions $w^{0}=v^{0}-q^{0}$ and $w^{1}=v^{1}-q^{1}$. As a consequence, using Lemma 2.1, we deduce that for $\tau>2$,

$$
\|v(\tau)-q(\tau)\|_{H_{L}^{1}(0,1)}^{2}+\left\|\partial_{t} v(\tau)-\partial_{t} q(\tau)\right\|_{L^{2}(0,1)}^{2} \leqslant \alpha\left(\left\|v^{0}-q^{0}\right\|_{H_{L}^{1}(0,1)}^{2}+\left\|v^{1}-q^{1}\right\|_{L^{2}(0,1)}^{2}\right) .
$$

Now let us set $\tilde{w}=v-q_{b}$ where $v$ and $q_{b}$ are respectively the solutions of (1) and (7). Then it can be easily checked that $\tilde{w}$ is the solution of

$$
\begin{cases}\partial_{t t} \tilde{w}-\partial_{x x} \tilde{w}=0 & \text { in }(0, \infty) \times(0,1), \\ \tilde{w}(t, 0)=0 & \text { for } t \in(0, \infty), \\ \left(\partial_{x} \tilde{w}-\partial_{t} \tilde{w}\right)(t, 1)=0 & \text { for } t \in(0, \infty), \\ \tilde{w}(\tau, x)=v(\tau, x)-q_{b}^{0}(x), \quad \partial_{t} \tilde{w}(\tau, x)=\partial_{t} v(\tau, x)-q_{b}^{1}(x) & \text { for } x \in(0,1) .\end{cases}
$$


Thus, $w(t, x):=\tilde{w}(\tau-t, x)$ satisfies $(16)$ with $w^{0}(x)=v(\tau, x)-q_{b}^{0}(x)$ and with $w^{1}(x)=-\partial_{t} v(\tau, x)+$ $q_{b}^{1}(x)$. As a consequence, using Lemma 2.1, we deduce that for $\tau>2$,

$$
\left\|v(0)-q_{b}(0)\right\|_{H_{L}^{1}(0,1)}^{2}+\left\|\partial_{t} v(0)-\partial_{t} q_{b}(0)\right\|_{L^{2}(0,1)}^{2} \leqslant \alpha\left(\left\|v(\tau)-q_{b}^{0}\right\|_{H_{L}^{1}(0,1)}^{2}+\left\|\partial_{t} v(\tau)-q_{b}^{1}\right\|_{L^{2}(0,1)}^{2}\right) .
$$

In particular for $\left(q_{b}^{0}, q_{b}^{1}\right)=\left(q(\tau), \partial_{t} q(\tau)\right)=F_{y}\left(q^{0}, q^{1}\right)$, we deduce from (25) and (27) that

$$
\left\|v^{0}-q_{b}(0)\right\|_{H_{L}^{1}(0,1)}^{2}+\left\|v^{1}-\partial_{t} q_{b}(0)\right\|_{L^{2}(0,1)}^{2} \leqslant \alpha^{2}\left(\left\|v^{0}-q^{0}\right\|_{H_{L}^{1}(0,1)}^{2}+\left\|v^{1}-q^{1}\right\|_{L^{2}(0,1)}^{2}\right) .
$$

Consequently, we deduce that

$$
\left\|\left(v^{0}, v^{1}\right)-B_{y} \circ F_{y}\left(q^{0}, q^{1}\right)\right\|_{H_{L}^{1}(0,1) \times L^{2}(0,1)}^{2} \leqslant \alpha^{2}\left\|\left(v^{0}, v^{1}\right)-\left(q^{0}, q^{1}\right)\right\|_{H_{L}^{1}(0,1) \times L^{2}(0,1)}^{2}
$$

and by induction, we deduce (9).

\section{Numerical analysis of a damped wave equation}

In order to prove Theorem 1.2, we first need, as in the theoretical part, to analyze a damped wave equation. More precisely, let us first consider the following finite-difference discretization of system (16) with the presence of a "pertubation" $\eta=\eta(t)$ at $x=1$ :

$$
\begin{cases}w_{j}^{\prime \prime}-\frac{w_{j+1}-2 w_{j}+w_{j-1}}{h^{2}}-\nu\left(w_{j+1}^{\prime}-2 w_{j}^{\prime}+w_{j-1}^{\prime}\right)=0 & \text { in }(0, \tau), 1 \leqslant j \leqslant N, \\ w_{0}=0 & \text { in }(0, \tau), \\ \frac{w_{N+1}-w_{N}}{h}+w_{N+1}^{\prime}=\eta & \text { in }(0, \tau), \\ w_{j}(0)=w_{j}^{0}, \quad w_{j}^{\prime}(0)=w_{j}^{1} & 0 \leqslant j \leqslant N+1 .\end{cases}
$$

The above system, with the add of the numerical viscosity term $-\nu\left(w_{j+1}^{\prime}-2 w_{j}^{\prime}+w_{j-1}^{\prime}\right)$ and in the case $\eta \equiv 0$, was studied in [36]. They show the uniform exponential stability of the above system. Here we prove a uniform decay of the energy with presence of the perturbation $\eta$.

Let us define the following energy associated to the system (29):

$$
\mathcal{E}_{h}(t)=\frac{h}{2} \sum_{j=0}^{N}\left[\left(w_{j}^{\prime}(t)\right)^{2}+\left(\frac{w_{j+1}(t)-w_{j}(t)}{h}\right)^{2}\right]+\frac{\nu h^{2}}{2}\left(w_{N+1}^{\prime}(t)\right)^{2}
$$

Lemma 3.1. The energy $\mathcal{E}_{h}$ defined by (30) satisfies

$$
\mathcal{E}_{h}^{\prime}=-\left(w_{N+1}^{\prime}\right)^{2}-h \nu \sum_{j=0}^{N}\left(w_{j+1}^{\prime}-w_{j}^{\prime}\right)^{2}+\eta w_{N+1}^{\prime}+h^{2} \nu \eta^{\prime} w_{N+1}^{\prime}
$$

in $(0, \infty)$.

Proof. In what follows, we use the classical formula (Abel transformation)

$$
\sum_{j=1}^{N}\left(b_{j}-b_{j-1}\right) a_{j}=b_{N} a_{N+1}-b_{0} a_{0}-\sum_{j=0}^{N} b_{j}\left(a_{j+1}-a_{j}\right)
$$


Applying (32), we deduce the following relations:

$$
\begin{aligned}
\sum_{j=1}^{N}\left(w_{j+1}-2 w_{j}+w_{j-1}\right) w_{j}^{\prime} & =\sum_{j=1}^{N}\left[\left(w_{j+1}-w_{j}\right)-\left(w_{j}-w_{j-1}\right)\right] w_{j}^{\prime} \\
& =\left(w_{N+1}-w_{N}\right) w_{N+1}^{\prime}-\left(w_{1}-w_{0}\right) w_{0}^{\prime}-\sum_{j=0}^{N}\left(w_{j+1}-w_{j}\right)\left(w_{j+1}^{\prime}-w_{j}^{\prime}\right),
\end{aligned}
$$

and

$$
\begin{aligned}
\sum_{j=1}^{N}\left(w_{j+1}^{\prime}-2 w_{j}^{\prime}+w_{j-1}^{\prime}\right) w_{j}^{\prime} & =\sum_{j=1}^{N}\left[\left(w_{j+1}^{\prime}-w_{j}^{\prime}\right)-\left(w_{j}^{\prime}-w_{j-1}^{\prime}\right)\right] w_{j}^{\prime} \\
& =\left(w_{N+1}^{\prime}-w_{N}^{\prime}\right) w_{N+1}^{\prime}-\left(w_{1}^{\prime}-w_{0}^{\prime}\right) w_{0}^{\prime}-\sum_{j=0}^{N}\left(w_{j+1}^{\prime}-w_{j}^{\prime}\right)^{2} .
\end{aligned}
$$

Then, we multiply the first equation of $(29)$ by $h w_{j}^{\prime}$, and by using the two above formulas, we obtain

$$
\begin{aligned}
\frac{h}{2} \sum_{j=1}^{N} \frac{d}{d t}\left(w_{j}^{\prime}\right)^{2}-\frac{w_{N+1}^{\prime}\left(w_{N+1}-w_{N}\right)}{h}+h \sum_{j=0}^{N} \frac{\left(w_{j+1}-w_{j}\right)\left(w_{j+1}^{\prime}-w_{j}^{\prime}\right)}{h^{2}} & \\
& -h \nu\left(w_{N+1}^{\prime}-w_{N}^{\prime}\right) w_{N+1}^{\prime}+h \nu \sum_{j=0}^{N}\left(w_{j+1}^{\prime}-w_{j}^{\prime}\right)^{2}=0
\end{aligned}
$$

The above equation, combined with the boundary condition in (29), yields

$$
\begin{aligned}
\frac{h}{2} \sum_{j=0}^{N} \frac{d}{d t}\left[\left(w_{j}^{\prime}\right)^{2}+\left(\frac{w_{j+1}-w_{j}}{h}\right)^{2}\right]+h \nu \sum_{j=0}^{N}\left(w_{j+1}^{\prime}-w_{j}^{\prime}\right)^{2} & +\left(w_{N+1}^{\prime}\right)^{2}-\eta w_{N+1}^{\prime} \\
& +\frac{1}{2} h^{2} \nu \frac{d}{d t}\left(w_{N+1}^{\prime}\right)^{2}-h^{2} \nu \eta^{\prime} w_{N+1}^{\prime}=0
\end{aligned}
$$

which implies (31).

The previous lemma shows that, without the "perturbation" $\eta$, the function $\mathcal{E}_{h}$ is a nonincreasing function of time. We improve this result in the following lemma.

Lemma 3.2. Assume $\tau>2$. Then there exist $\beta \in(0,1)$ and $C=C(\nu)$ such that

$$
\mathcal{E}_{h}(\tau) \leqslant \beta \mathcal{E}_{h}(0)+C \int_{0}^{\tau}\left[\eta^{2}+h^{2}\left(\eta^{\prime}\right)^{2}\right] d s
$$

Proof. Let us define the following function of time

$$
W_{\varepsilon}:=\mathcal{E}_{h}+\varepsilon \sum_{j=1}^{N} w_{j}^{\prime} j h \frac{w_{j+1}-w_{j-1}}{2}-\frac{\varepsilon h}{4}\left(w_{N+1}^{\prime}\right)^{2} .
$$


From (31) and the first equation of (29), we have

$$
\begin{aligned}
& W_{\varepsilon}^{\prime}=-\left(w_{N+1}^{\prime}\right)^{2}-h \nu \sum_{j=0}^{N}\left(w_{j+1}^{\prime}-w_{j}^{\prime}\right)^{2}+\eta w_{N+1}^{\prime}+h^{2} \nu \eta^{\prime} w_{N+1}^{\prime} \\
&+\varepsilon \sum_{j=1}^{N} \frac{w_{j+1}-2 w_{j}+w_{j-1}}{h^{2}} j h \frac{w_{j+1}-w_{j-1}}{2}+\varepsilon \sum_{j=1}^{N} \nu\left(w_{j+1}^{\prime}-2 w_{j}^{\prime}+w_{j-1}^{\prime}\right) j h \frac{w_{j+1}-w_{j-1}}{2} \\
&+\varepsilon \sum_{j=1}^{N} w_{j}^{\prime} j h \frac{w_{j+1}^{\prime}-w_{j-1}^{\prime}}{2}-\frac{\varepsilon h}{2} w_{N+1}^{\prime} w_{N+1}^{\prime \prime} .
\end{aligned}
$$

First, using (32) and the third equation of (29), one obtains

$$
\begin{aligned}
\varepsilon \sum_{j=1}^{N} \frac{w_{j+1}-2 w_{j}+w_{j-1}}{h^{2}} j h\left(\frac{w_{j+1}-w_{j-1}}{2}\right) & =\frac{\varepsilon}{2 h} \sum_{j=1}^{N} j\left(\left[w_{j+1}-w_{j}\right]^{2}-\left[w_{j}-w_{j-1}\right]^{2}\right) \\
& =\frac{\varepsilon}{2}\left(\frac{w_{N+1}-w_{N}}{h}\right)^{2}-\varepsilon \frac{h}{2} \sum_{j=0}^{N}\left(\frac{w_{j+1}-w_{j}}{h}\right)^{2} \\
& =\frac{\varepsilon}{2}\left(\eta-w_{N+1}^{\prime}\right)^{2}-\varepsilon \frac{h}{2} \sum_{j=0}^{N}\left(\frac{w_{j+1}-w_{j}}{h}\right)^{2}
\end{aligned}
$$

Second, we have

$$
\begin{aligned}
& \varepsilon \nu h \sum_{j=1}^{N}\left(w_{j+1}^{\prime}-2 w_{j}^{\prime}+w_{j-1}^{\prime}\right) j\left(\frac{w_{j+1}-w_{j-1}}{2}\right) \\
= & \varepsilon \nu h \frac{1}{2} \sum_{j=1}^{N}\left(w_{j+1}^{\prime}-2 w_{j}^{\prime}+w_{j-1}^{\prime}\right) j\left(w_{j+1}-w_{j}\right)+\varepsilon \nu h \frac{1}{2} \sum_{j=1}^{N}\left(w_{j+1}^{\prime}-2 w_{j}^{\prime}+w_{j-1}^{\prime}\right) j\left(w_{j}-w_{j-1}\right) \\
= & \varepsilon \nu h \frac{1}{2} \sum_{j=1}^{N}\left(w_{j+1}^{\prime}-w_{j}^{\prime}\right) j\left(w_{j+1}-w_{j}\right)-\varepsilon \nu h \frac{1}{2} \sum_{j=1}^{N}\left(w_{j}^{\prime}-w_{j-1}^{\prime}\right) j\left(w_{j+1}-w_{j}\right) \\
& +\varepsilon \nu h \frac{1}{2} \sum_{j=1}^{N}\left(w_{j+1}^{\prime}-w_{j}^{\prime}\right) j\left(w_{j}-w_{j-1}\right)-\varepsilon \nu h \frac{1}{2} \sum_{j=1}^{N}\left(w_{j}^{\prime}-w_{j-1}^{\prime}\right) j\left(w_{j}-w_{j-1}\right) .
\end{aligned}
$$

We have

$$
\left|\varepsilon \nu h \frac{1}{2} \sum_{j=1}^{N}\left(w_{j+1}^{\prime}-w_{j}^{\prime}\right) j\left(w_{j+1}-w_{j}\right)\right| \leqslant \frac{\nu h}{8} \sum_{j=1}^{N}\left(w_{j+1}^{\prime}-w_{j}^{\prime}\right)^{2}+\frac{\nu h}{2} \sum_{j=1}^{N} \varepsilon^{2}\left(\frac{w_{j+1}-w_{j}}{h}\right)^{2}
$$

and we compute similarly the three other terms of (37) to obtain

$$
\begin{array}{r}
\varepsilon \nu h \sum_{j=1}^{N}\left(w_{j+1}^{\prime}-2 w_{j}^{\prime}+w_{j-1}^{\prime}\right) j\left(\frac{w_{j+1}-w_{j-1}}{2}\right) \\
\leqslant \frac{\nu h}{2} \sum_{j=0}^{N}\left(w_{j+1}^{\prime}-w_{j}^{\prime}\right)^{2}+4 \nu \varepsilon^{2} \frac{h}{2} \sum_{j=0}^{N}\left(\frac{w_{j+1}-w_{j}}{h}\right)^{2} .
\end{array}
$$


Finally, standard computations (and again the third equation of (29)) yield

$$
\begin{aligned}
\varepsilon \frac{1}{2} \sum_{j=1}^{N} w_{j}^{\prime} j h\left(w_{j+1}^{\prime}-w_{j-1}^{\prime}\right)= & \varepsilon \frac{1}{2} \sum_{j=1}^{N} w_{j}^{\prime} j h w_{j+1}^{\prime}-\varepsilon \frac{1}{2} \sum_{j=1}^{N} w_{j}^{\prime} j h w_{j-1}^{\prime} \\
= & -\varepsilon \frac{h}{2} \sum_{j=0}^{N} w_{j}^{\prime} w_{j+1}^{\prime}+\frac{\varepsilon}{2} w_{N}^{\prime} w_{N+1}^{\prime} \\
= & -\varepsilon \frac{h}{2} \sum_{j=0}^{N}\left(w_{j}^{\prime}\right)^{2}+\varepsilon \frac{h}{2} \sum_{j=0}^{N} w_{j}^{\prime}\left(w_{j}^{\prime}-w_{j+1}^{\prime}\right)+\frac{\varepsilon}{2}\left(w_{N+1}^{\prime}\right)^{2} \\
& +\frac{\varepsilon h}{2} w_{N+1}^{\prime} w_{N+1}^{\prime \prime}-\frac{\varepsilon h}{2} \eta^{\prime} w_{N+1}^{\prime} .
\end{aligned}
$$

On the other hand,

$$
\left|\varepsilon \frac{h}{2} \sum_{j=0}^{N} w_{j}^{\prime}\left(w_{j}^{\prime}-w_{j+1}^{\prime}\right)\right| \leqslant \frac{1}{2} \nu h \sum_{j=0}^{N}\left(w_{j}^{\prime}-w_{j+1}^{\prime}\right)^{2}+\frac{\varepsilon^{2} h}{8 \nu} \sum_{j=0}^{N}\left(w_{j}^{\prime}\right)^{2} .
$$

Gathering (35), (36), (38), (39) and the above equation yields

$$
\begin{aligned}
W_{\varepsilon}^{\prime} \leqslant-\left(w_{N+1}^{\prime}\right)^{2}-h \nu & \sum_{j=0}^{N}\left(w_{j+1}^{\prime}-w_{j}^{\prime}\right)^{2}+\eta w_{N+1}^{\prime}+h^{2} \nu \eta^{\prime} w_{N+1}^{\prime} \\
- & \varepsilon \frac{h}{2} \sum_{j=0}^{N}\left(\frac{w_{j+1}-w_{j}}{h}\right)^{2}+\frac{\varepsilon}{2}\left(w_{N+1}^{\prime}\right)^{2}+\frac{\varepsilon \eta^{2}}{2}-\varepsilon \eta w_{N+1}^{\prime} \\
+ & \frac{\nu h}{2} \sum_{j=0}^{N}\left(w_{j+1}^{\prime}-w_{j}^{\prime}\right)^{2}+4 \nu \varepsilon^{2} \frac{h}{2} \sum_{j=0}^{N}\left(\frac{w_{j+1}-w_{j}}{h}\right)^{2} \\
-\varepsilon \frac{h}{2} \sum_{j=0}^{N}\left(w_{j}^{\prime}\right)^{2}+\frac{\varepsilon}{2}\left(w_{N+1}^{\prime}\right)^{2}-\frac{\varepsilon h}{2} \eta^{\prime} w_{N+1}^{\prime} & +\frac{1}{2} \nu h \sum_{j=0}^{N}\left(w_{j}^{\prime}-w_{j+1}^{\prime}\right)^{2}+\frac{\varepsilon^{2} h}{8 \nu} \sum_{j=0}^{N}\left(w_{j}^{\prime}\right)^{2} .
\end{aligned}
$$

The above relation and the definition (30) imply

$$
\begin{array}{r}
W_{\varepsilon}^{\prime} \leqslant\left(\varepsilon\left(1+\frac{\nu h^{2}}{2}\right)-1\right)\left(w_{N+1}^{\prime}\right)^{2}-\varepsilon \mathcal{E}_{h}(t)+\varepsilon^{2}\left(4 \nu+\frac{1}{4 \nu}\right) \mathcal{E}_{h}(t)-\varepsilon^{2}\left(4 \nu+\frac{1}{4 \nu}\right) \frac{\nu h^{2}}{2}\left(w_{N+1}^{\prime}\right)^{2} \\
+\eta w_{N+1}^{\prime}+h^{2} \nu \eta^{\prime} w_{N+1}^{\prime}+\varepsilon\left(\frac{\eta^{2}}{2}-\eta w_{N+1}^{\prime}-\frac{h}{2} \eta^{\prime} w_{N+1}^{\prime}\right),
\end{array}
$$

and thus

$$
\begin{array}{r}
W_{\varepsilon}^{\prime} \leqslant\left(-\varepsilon^{2}\left(4 \nu+\frac{1}{4 \nu}\right) \frac{\nu h^{2}}{2}+\varepsilon\left(2+\frac{\nu h^{2}}{2}\right)-\frac{1}{4}\right)\left(w_{N+1}^{\prime}\right)^{2}-\varepsilon \mathcal{E}_{h}(t)+\varepsilon^{2}\left(4 \nu+\frac{1}{4 \nu}\right) \mathcal{E}_{h}(t) \\
+(1+2 \varepsilon) \eta^{2}+\left(h^{4} \nu^{2}+\varepsilon^{2} h^{2}\right)\left(\eta^{\prime}\right)^{2} .
\end{array}
$$

In particular, there exists $\varepsilon_{0}=\varepsilon_{0}(\nu)$ uniform in $h$ such that for $\varepsilon \in\left(0, \varepsilon_{0}\right)$, we have

$$
W_{\varepsilon}^{\prime} \leqslant-\varepsilon \mathcal{E}_{h}+\varepsilon^{2}\left(4 \nu+\frac{1}{4 \nu}\right) \mathcal{E}_{h}+(1+2 \varepsilon) \eta^{2}+\left(h^{4} \nu^{2}+\varepsilon^{2} h^{2}\right)\left(\eta^{\prime}\right)^{2} .
$$


On the other hand, we have

$$
\begin{gathered}
\sum_{j=1}^{N} w_{j}^{\prime} j h \frac{w_{j+1}-w_{j-1}}{2}=\sum_{j=1}^{N} w_{j}^{\prime} j h \frac{h}{2}\left(\frac{w_{j+1}-w_{j}}{h}+\frac{w_{j}-w_{j-1}}{h}\right) \\
\left|\sum_{j=1}^{N} w_{j}^{\prime} j h \frac{w_{j+1}-w_{j-1}}{2}\right| \\
\leqslant \frac{h}{2} \sum_{j=0}^{N}\left(w_{j}^{\prime}\right)^{2}+\frac{h}{2} \sum_{j=0}^{N-1}\left(\frac{w_{j+1}-w_{j}}{h}\right)^{2}+\frac{h}{4}\left(\frac{w_{N+1}-w_{N}}{h}\right)^{2} \\
\leqslant \mathcal{E}_{h}-\frac{\nu h^{2}}{2}\left(w_{N+1}^{\prime}\right)^{2}
\end{gathered}
$$

and thus

$$
W_{\varepsilon} \leqslant \mathcal{E}_{h}+\varepsilon \mathcal{E}_{h}-\varepsilon \frac{\nu h^{2}}{2}\left(w_{N+1}^{\prime}\right)^{2}-\frac{\varepsilon h}{4}\left(w_{N+1}^{\prime}\right)^{2} \leqslant(1+\varepsilon) \mathcal{E}_{h} .
$$

Gathering (41) and (43) and taking $\varepsilon \in\left(0, \varepsilon_{0}\right)$, we get

$$
W_{\varepsilon}^{\prime} \leqslant \frac{\left(-\varepsilon+\varepsilon^{2}\left(4 \nu+\frac{1}{4 \nu}\right)\right.}{1+\varepsilon} W_{\varepsilon}+(1+2 \varepsilon) \eta^{2}+\left(h^{4} \nu^{2}+\varepsilon^{2} h^{2}\right)\left(\eta^{\prime}\right)^{2} .
$$

The above equation implies

$$
W_{\varepsilon}(t) \leqslant \exp \left(\frac{-\varepsilon+\varepsilon^{2}\left(4 \nu+\frac{1}{4 \nu}\right)}{1+\varepsilon} t\right) W_{\varepsilon}(0)+\int_{0}^{t}\left[(1+2 \varepsilon) \eta^{2}+\left(h^{4} \nu^{2}+\varepsilon^{2} h^{2}\right)\left(\eta^{\prime}\right)^{2}\right] d s .
$$

In order to estimate from below $W_{\varepsilon}$ (in function of $\mathcal{E}_{h}$ ), we use the third equation of (29) to obtain

$$
\frac{\varepsilon h}{4}\left(w_{N+1}^{\prime}\right)^{2} \leqslant \frac{\varepsilon h}{4}\left[(1+\varepsilon)\left(\frac{w_{N+1}-w_{N}}{h}\right)^{2}+\left(1+\frac{1}{\varepsilon}\right) \eta^{2}\right] .
$$

We deduce from (42) and from the above equation that

$$
\begin{aligned}
W_{\varepsilon} \geqslant \mathcal{E}_{h}-\varepsilon\left[\frac{h}{2} \sum_{j=0}^{N}\left(w_{j}^{\prime}\right)^{2}+\frac{h}{2} \sum_{j=0}^{N-1}\left(\frac{w_{j+1}-w_{j}}{h}\right)^{2}+\right. & \left.\frac{h}{4}\left(\frac{w_{N+1}-w_{N}}{h}\right)^{2}\right] \\
& -\frac{\varepsilon h}{4}\left[(1+\varepsilon)\left(\frac{w_{N+1}-w_{N}}{h}\right)^{2}+\left(1+\frac{1}{\varepsilon}\right) \eta^{2}\right]
\end{aligned}
$$

and thus

$$
W_{\varepsilon} \geqslant \mathcal{E}_{h}-\varepsilon \mathcal{E}_{h}-\frac{\varepsilon^{2}}{2} \mathcal{E}_{h}-\frac{h}{4}(\varepsilon+1) \eta^{2} .
$$

Combining the above equation with (43) and (44) yields

$$
\begin{aligned}
\left(1-\varepsilon-\frac{\varepsilon^{2}}{2}\right) \mathcal{E}_{h}(t) \leqslant(1+\varepsilon) \exp \left(\frac{-\varepsilon+\varepsilon^{2}\left(4 \nu+\frac{1}{4 \nu}\right)}{1+\varepsilon} t\right) & \mathcal{E}_{h}(0) \\
& +\frac{(\varepsilon+1) h}{4} \eta^{2}(t)+\int_{0}^{t}\left[(1+2 \varepsilon) \eta^{2}+\left(h^{4} \nu^{2}+\varepsilon^{2} h^{2}\right)\left(\eta^{\prime}\right)^{2}\right] d s .
\end{aligned}
$$


and thus

$$
\begin{aligned}
\mathcal{E}_{h}(t) \leqslant \frac{(1+\varepsilon)}{\left(1-\varepsilon-\frac{\varepsilon^{2}}{2}\right)} \exp \left(\frac{-\varepsilon+\varepsilon^{2}\left(4 \nu+\frac{1}{4 \nu}\right)}{1+\varepsilon} t\right) & \mathcal{E}_{h}(0) \\
& +\frac{(\varepsilon+1) h}{4\left(1-\varepsilon-\frac{\varepsilon^{2}}{2}\right)} \eta^{2}(t)+\int_{0}^{t} \frac{\left[(1+2 \varepsilon) \eta^{2}+\left(h^{4} \nu^{2}+\varepsilon^{2} h^{2}\right)\left(\eta^{\prime}\right)^{2}\right]}{\left(1-\varepsilon-\frac{\varepsilon^{2}}{2}\right)} d s .
\end{aligned}
$$

Using that

$$
\eta^{2}(t) \leqslant\left(\frac{1}{t}+\frac{1}{h}\right) \int_{0}^{t} \eta^{2} d s+h \int_{0}^{t}\left(\eta^{\prime}\right)^{2} d s,
$$

we deduce that there exists a constant $C=C\left(t, \nu, \varepsilon_{0}\right)$ such that for $\varepsilon \in\left(0, \varepsilon_{0}\right)$,

$$
\mathcal{E}_{h}(t) \leqslant \frac{(1+\varepsilon)}{\left(1-\varepsilon-\frac{\varepsilon^{2}}{2}\right)} \exp \left(\frac{-\varepsilon+\varepsilon^{2}\left(4 \nu+\frac{1}{4 \nu}\right)}{1+\varepsilon} t\right) \mathcal{E}_{h}(0)+C \int_{0}^{t}\left[\eta^{2}+h^{2}\left(\eta^{\prime}\right)^{2}\right] d s .
$$

Consequently, if we set

$$
f_{\nu}(\varepsilon):=\left(\frac{1+\varepsilon}{\varepsilon+\varepsilon^{2}\left(4 \nu+\frac{1}{4 \nu}\right)}\right) \ln \frac{(1+\varepsilon)}{\left(1-\varepsilon-\frac{\varepsilon^{2}}{2}\right)}
$$

then, for $\tau>f_{\nu}(\varepsilon)$, we have

$$
\mathcal{E}_{h}(\tau) \leqslant \beta \mathcal{E}_{h}(0)+C \int_{0}^{\tau}\left[\eta^{2}+h^{2}\left(\eta^{\prime}\right)^{2}\right] d s
$$

with

$$
\beta:=\frac{(1+\varepsilon)}{\left(1-\varepsilon-\frac{\varepsilon^{2}}{2}\right)} \exp \left(\frac{-\varepsilon+\varepsilon^{2}\left(4 \nu+\frac{1}{4 \nu}\right)}{1+\varepsilon} \tau\right) \in(0,1) .
$$

Since $\lim _{\varepsilon \rightarrow 0^{+}} f_{\nu}(\varepsilon)=2$, we conclude the proof of the lemma.

\section{Numerical schemes for (1) with vanishing viscosity}

In this section, we consider two numerical approximations of (1):

$$
\begin{cases}v_{j}^{\prime \prime}-\frac{v_{j+1}-2 v_{j}+v_{j-1}}{h^{2}}-\nu\left(v_{j+1}^{\prime}-2 v_{j}^{\prime}+v_{j-1}^{\prime}\right)=0 & \text { in }(0, \tau), 1 \leqslant j \leqslant N, \\ v_{0}=0 & \text { in }(0, \tau), \\ v_{N+1}=v_{N} & \text { in }(0, \tau), \\ v_{j}(0)=v_{j}^{0}, \quad v_{j}^{\prime}(0)=v_{j}^{1} & 0 \leqslant j \leqslant N+1,\end{cases}
$$

and

$$
\begin{cases}v_{b, j}^{\prime \prime}-\frac{v_{b, j+1}-2 v_{b, j}+v_{b, j-1}}{h^{2}}+\nu\left(v_{b, j+1}^{\prime}-2 v_{b, j}^{\prime}+v_{b, j-1}^{\prime}\right)=0 & \text { in }(0, \tau), 1 \leqslant j \leqslant N \\ v_{b, 0}=0 & \text { in }(0, \tau), \\ v_{b, N+1}=v_{b, N} & \text { in }(0, \tau), \\ v_{b, j}(\tau)=v_{b, j}^{0}, \quad v_{b, j}^{\prime}(\tau)=v_{b, j}^{1} & 0 \leqslant j \leqslant N+1 .\end{cases}
$$


Remark 4.1. We need two different approximations of (1) since we add a numerical viscosity in these numerical schemes and since there is a change of sign between the forward and the backward systems. The idea to prove Theorem 1.2 is to make the differences between theses systems and systems (12) and (14) (respectively) and to reduce to the system (29) studied in the previous subsection.

We recall first some results of convergence (proved in [36]) of these numerical schemes. Then we consider a numerical property associated to the admissibility of the observation operator considered here.

\subsection{Convergence of numerical schemes with vanishing viscosity}

Here we recall results proved in [36] of systems (12) and (14).

Let us define

$$
E_{h}\left(a_{h}, b_{h}\right):=\frac{h}{2} \sum_{j=0}^{N}\left[b_{j}^{2}+\left(\frac{a_{j+1}-a_{j}}{h}\right)^{2}\right] \quad\left(a_{h}=\left(a_{j}\right)_{0 \leqslant j \leqslant N+1}, b_{h}=\left(b_{j}\right)_{0 \leqslant j \leqslant N+1} \in \mathbb{R}^{N+2}\right) .
$$

Let us also consider the operator

$$
P_{h}(a):=(a(j h))_{0 \leqslant j \leqslant N+1},
$$

for any function $a$ (regular enough).

It is proved in [36] that if $v_{h}:=\left(v_{j}\right)_{0 \leqslant j \leqslant N+1}$ is the solution of (46), if $v$ is the solution of (1) and if $\mathcal{E}_{h}(0)$ (defined in (30)) converges towards $E(0)$ (defined in (19)) as $h \rightarrow 0$ then

$$
\begin{gathered}
E_{h}\left(P_{h}(v(\tau))-v_{h}(\tau), P_{h}\left(\partial_{t} v(\tau)\right)-v_{h}^{\prime}(\tau)\right) \rightarrow 0, \\
\frac{\nu h^{2}}{2}\left(\partial_{t} v(\tau, 1)-v_{N+1}^{\prime}(\tau)\right)^{2} \rightarrow 0 \\
\int_{0}^{\tau}\left[\left(v_{N+1}^{\prime}-\partial_{t} v(1)\right)^{2}\right] d s \rightarrow 0,
\end{gathered}
$$

as $h \rightarrow 0$. In particular,

$$
\mathcal{E}_{h}(\tau) \rightarrow E(\tau) \quad \text { as } h \rightarrow 0
$$

We can note that $\tilde{v}$ defined by

$$
\tilde{v}(t, x):=v(\tau-t, x)
$$

is solution of

$$
\begin{cases}\partial_{t t} \tilde{v}-\partial_{x x} \tilde{v}=0 & \text { in }(0, \tau) \times(0,1), \\ \tilde{v}(t, 0)=0 & \text { for } t \in(0, \tau), \\ \partial_{x} \tilde{v}(t, 1)=0 & \text { for } t \in(0, \tau), \\ \tilde{v}(0, x)=v(\tau, x), \quad \partial_{t} \tilde{v}(0, x)=-\partial_{t} v(\tau, x) & \text { for } x \in(0,1) .\end{cases}
$$

One can check that $\tilde{v}(\tau)=v^{0}, \partial_{t} \tilde{v}(\tau)=-v^{1}$.

Moreover, if we set

$$
\tilde{v}_{b, j}(t):=v_{b, j}(\tau-t) \quad(0 \leqslant j \leqslant N+1, t \in(0, \tau)),
$$

where $v_{b, h}:=\left(v_{b, j}\right)_{0 \leqslant j \leqslant N+1}$ is solution of $(47)$ then

$$
\begin{cases}\tilde{v}_{b, j}^{\prime \prime}-\frac{\tilde{v}_{b, j+1}-2 \tilde{v}_{b, j}+\tilde{v}_{b, j-1}}{h^{2}}-\nu\left(\tilde{v}_{b, j+1}^{\prime}-2 \tilde{v}_{b, j}^{\prime}+\tilde{v}_{b, j-1}^{\prime}\right)=0 & \text { in }(0, \tau), 1 \leqslant j \leqslant N, \\ \tilde{v}_{b, 0}=0 & \text { in }(0, \tau), \\ \tilde{v}_{b, N+1}=\tilde{v}_{b, N} & \text { in }(0, \tau), \\ \tilde{v}_{b, j}(0)=v_{b, j}^{0}, \quad \tilde{v}_{b, j}^{\prime}(0)=-v_{b, j}^{1} & 0 \leqslant j \leqslant N+1 .\end{cases}
$$


One can check $\tilde{v}_{b, h}(\tau)=v_{b, h}(0)$ and $\tilde{v}_{b, h}^{\prime}(\tau)=-v_{b, h}^{\prime}(0)$.

Consequently, from (52) and from the result of convergence of [36], we deduce that

$$
\begin{gathered}
E_{h}\left(P_{h}\left(v^{0}\right)-v_{b, h}(0), P_{h}\left(v^{1}\right)-v_{b, h}^{\prime}(0)\right) \rightarrow 0, \\
\frac{\nu h^{2}}{2}\left(v^{1}(1)-v_{b, N+1}^{\prime}(0)\right)^{2} \rightarrow 0, \\
\int_{0}^{\tau}\left[\left(v_{b, N+1}^{\prime}-\partial_{t} v(1)\right)^{2}\right] d s \rightarrow 0 .
\end{gathered}
$$

\subsection{Numerical admissibility}

In this section, we show the numerical admissibility of the observation operator. This is the numerical counterpart of the admissibility result (or hidden regularity) for the system (1) for systems (12) and (14):

$$
\int_{0}^{\tau}\left|\partial_{t} v(t, 1)\right|^{2} d t \leqslant C E(0)
$$

Lemma 4.2. Assume that $v_{h}=\left(v_{j}\right)_{0 \leqslant j \leqslant N+1}$ is solution of (46). Then there exists a constant $C=$ $C(\tau)$ independent of $h$ such that

$$
\int_{0}^{\tau}\left(v_{N+1}^{\prime}\right)^{2} d t \leqslant C \frac{h}{2} \sum_{j=0}^{N}\left[\left(v_{j}^{1}\right)^{2}+\left(\frac{v_{j+1}^{0}-v_{j}^{0}}{h}\right)^{2}\right] .
$$

In the above result, it is important to notice that the constant $C$ is independent of $h$. Indeed, for a fixed $h$, since we deal with a finite dimensional system, the observation operator is admissible and thus (58) holds for a constant $C=C(h)$. In the proof of the main result (Section 5), we need the admissibility estimate (58) for a constant $C$ independent of $h$.

Before proving Lemma 4.2, let us show how to deduce from it similar inequalities for $v_{h}^{\prime}$ and $v_{b, h}^{\prime}$. Since $v_{h}^{\prime}$ is also solution of (46) if $v_{h}$ is solution of (46) (but with different initial conditions), then we can apply Lemma 4.2 to $v_{h}^{\prime}$ and we obtain

$$
\int_{0}^{\tau}\left(v_{N+1}^{\prime \prime}\right)^{2} d t \leqslant C \frac{h}{2}\left[\sum_{j=1}^{N}\left(\frac{v_{j+1}^{0}-2 v_{j}^{0}+v_{j-1}^{0}}{h^{2}}+\nu\left(v_{j+1}^{1}-2 v_{j}^{1}+v_{j-1}^{1}\right)\right)^{2}+\sum_{j=0}^{N}\left(\frac{v_{j+1}^{1}-v_{j}^{1}}{h}\right)^{2}\right]
$$

and thus

$$
\int_{0}^{\tau}\left(v_{N+1}^{\prime \prime}\right)^{2} d t \leqslant C \frac{h}{2}\left[\sum_{j=1}^{N}\left(\frac{v_{j+1}^{0}-2 v_{j}^{0}+v_{j-1}^{0}}{h^{2}}\right)^{2}+\sum_{j=0}^{N}\left(\frac{v_{j+1}^{1}-v_{j}^{1}}{h}\right)^{2}\right] .
$$

In particular, if $v^{0} \in H^{2}(0,1), v^{1} \in H^{1}(0,1)$, and if $v_{h}^{0}=P_{h}\left(v^{0}\right), v_{h}^{1}=P_{h}\left(v^{1}\right)$, then

$$
\int_{0}^{\tau}\left(v_{N+1}^{\prime \prime}\right)^{2} d t \leqslant C\left(\left\|v^{0}\right\|_{H^{2}(0,1)}^{2}+\left\|v^{1}\right\|_{H^{1}(0,1)}^{2}\right)
$$

where $C$ is a positive constant independent of $h$. Moreover, if $v_{b, h}$ is the solution of (47) with $v_{b, h}^{0}=$ $v_{h}(\tau)$ and $v_{b, h}^{1}=v_{h}^{\prime}(\tau)$, we deduce from (59) that

$$
\int_{0}^{\tau}\left(v_{b, N+1}^{\prime \prime}\right)^{2} d t \leqslant C \frac{h}{2}\left[\sum_{j=1}^{N}\left(\frac{v_{j+1}(\tau)-2 v_{j}(\tau)+v_{j-1}(\tau)}{h^{2}}\right)^{2}+\sum_{j=0}^{N}\left(\frac{v_{j+1}^{\prime}(\tau)-v_{j}^{\prime}(\tau)}{h}\right)^{2}\right] .
$$

Combining the above estimate with Lemma 4.3 below we deduce that

$$
\int_{0}^{\tau}\left(v_{b, N+1}^{\prime \prime}\right)^{2} d t \leqslant C\left(\left\|v^{0}\right\|_{H^{2}(0,1)}^{2}+\left\|v^{1}\right\|_{H^{1}(0,1)}^{2}\right) .
$$


Proof of Lemma 4.2. Let us multiply the first equation of (46) by $h v_{j}^{\prime}$. Following the proof of Lemma 3.1 , we obtain

$$
\frac{d}{d t}\left(\frac{h}{2} \sum_{j=0}^{N}\left[\left(v_{j}^{\prime}(t)\right)^{2}+\left(\frac{v_{j+1}(t)-v_{j}(t)}{h}\right)^{2}\right]\right) \leqslant 0
$$

Now let us multiply the first equation of (46) by $j h\left(v_{j+1}-v_{j-1}\right) / 2$. Using (32), we deduce

$$
\sum_{j=1}^{N} \frac{v_{j+1}-2 v_{j}+v_{j-1}}{h^{2}} j h\left(\frac{v_{j+1}-v_{j-1}}{2}\right)=-\frac{h}{2} \sum_{j=0}^{N}\left(\frac{v_{j+1}-v_{j}}{h}\right)^{2} .
$$

We also obtain, in similar way as (38), that

$$
\nu h \sum_{j=1}^{N}\left(v_{j+1}^{\prime}-2 v_{j}^{\prime}+v_{j-1}^{\prime}\right) j\left(\frac{v_{j+1}-v_{j-1}}{2}\right) \leqslant \frac{3 \nu h}{2}\left(\sum_{j=0}^{N}\left(v_{j}^{\prime}\right)^{2}+\sum_{j=0}^{N}\left(\frac{v_{j+1}-v_{j}}{h}\right)^{2}\right) .
$$

Finally, standard calculations yield

$$
\begin{aligned}
& \int_{0}^{\tau} \sum_{j=1}^{N} v_{j}^{\prime \prime}(j h) \frac{v_{j+1}-v_{j-1}}{2} d t \\
= & \sum_{j=1}^{N}\left(v_{j}^{\prime}(\tau)(j h) \frac{v_{j+1}(\tau)-v_{j-1}(\tau)}{2}-v_{j}^{\prime}(0)(j h) \frac{v_{j+1}(0)-v_{j-1}(0)}{2}\right)-\int_{0}^{\tau} \sum_{j=1}^{N} v_{j}^{\prime}(j h) \frac{v_{j+1}^{\prime}-v_{j-1}^{\prime}}{2} d t .
\end{aligned}
$$

Moreover,

$$
\sum_{j=1}^{N} v_{j}^{\prime}(j h)\left(v_{j+1}^{\prime}-v_{j-1}^{\prime}\right)=\left(v_{N+1}^{\prime}\right)^{2}-h \sum_{j=1}^{N} v_{j}^{\prime} v_{j+1}^{\prime} .
$$

Gathering the above equation, (63), (64) and (65) and using (62), we deduce (58).

Lemma 4.3. Assume that $v_{h}=\left(v_{j}\right)_{0 \leqslant j \leqslant N+1}$ is solution of (46). Then there exists a constant $C$ independent of $h$ such that

$$
\begin{aligned}
\frac{h}{2} \sum_{j=1}^{N}\left(\frac{v_{j+1}(t)-2 v_{j}(t)+v_{j-1}(t)}{h^{2}}\right)^{2}+ & \frac{h}{2} \sum_{j=0}^{N}\left(\frac{v_{j+1}^{\prime}(t)-v_{j}^{\prime}(t)}{h}\right)^{2} \\
& \leqslant C \frac{h}{2}\left[\sum_{j=1}^{N}\left(\frac{v_{j+1}^{0}-2 v_{j}^{0}+v_{j-1}^{0}}{h^{2}}\right)^{2}+\sum_{j=0}^{N}\left(\frac{v_{j+1}^{1}-v_{j}^{1}}{h}\right)^{2}\right] .
\end{aligned}
$$

Proof. Since $v_{h}^{\prime}$ is solution of (46), we can use (62) to obtain

$$
\frac{h}{2} \sum_{j=0}^{N}\left[\left(v_{j}^{\prime \prime}(t)\right)^{2}+\left(\frac{v_{j+1}^{\prime}(t)-v_{j}^{\prime}(t)}{h}\right)^{2}\right] \leqslant \frac{h}{2} \sum_{j=0}^{N}\left[\left(v_{j}^{\prime \prime}(0)\right)^{2}+\left(\frac{v_{j+1}^{\prime}(0)-v_{j}^{\prime}(0)}{h}\right)^{2}\right] .
$$

Moreover, $v_{0}^{\prime \prime}(t)=0$ and for $j=1, \cdots, N$,

$$
v_{j}^{\prime \prime}(t)=\frac{v_{j+1}-2 v_{j}+v_{j-1}}{h^{2}}+\nu\left(v_{j+1}^{\prime}-2 v_{j}^{\prime}+v_{j-1}^{\prime}\right)
$$


and thus

$$
\begin{aligned}
\sum_{j=1}^{N}\left(\frac{v_{j+1}-2 v_{j}+v_{j-1}}{h^{2}}\right)^{2} & \leqslant 2 \sum_{j=0}^{N}\left(v_{j}^{\prime \prime}(t)\right)^{2}+2 \sum_{j=1}^{N} \nu^{2}\left(v_{j+1}^{\prime}-2 v_{j}^{\prime}+v_{j-1}^{\prime}\right)^{2} \\
& \leqslant C \sum_{j=0}^{N}\left[\left(v_{j}^{\prime \prime}(t)\right)^{2}+\left(\frac{v_{j+1}^{\prime}(t)-v_{j}^{\prime}(t)}{h}\right)^{2}\right]
\end{aligned}
$$

In a similar way,

$$
\sum_{j=0}^{N}\left(v_{j}^{\prime \prime}(0)\right)^{2} \leqslant C\left[\sum_{j=1}^{N}\left(\frac{v_{j+1}(0)-2 v_{j}(0)+v_{j-1}(0)}{h^{2}}\right)^{2}+\sum_{j=0}^{N}\left(\frac{v_{j+1}^{\prime}(0)-v_{j}^{\prime}(0)}{h}\right)^{2}\right] .
$$

Combining the above equation with (66) and (67), we conclude the proof of the lemma.

\section{Proof of the main result}

This section is devoted to the proof of Theorem 1.2.

Proof of Theorem 1.2. Using the same arguments as in Proposition 1.1, let us define $w_{h}:=v_{h}-q_{h}$, where $v_{h}$ and $q_{h}$ are respectively the solutions of (46) and (12). It is easy to see that $w_{h}=\left(w_{j}\right)_{0 \leqslant j \leqslant N+1}$ satisfies (29) with the initial conditions $w_{j}^{0}=v_{j}^{0}-q_{j}^{0}$ and $w_{j}^{1}=v_{j}^{1}-q_{j}^{1}$, for $0 \leqslant j \leqslant N+1$ and with

$$
\eta:=\left(v_{N+1}^{\prime}-y\right) \text {. }
$$

From Lemma 3.2, we obtain the following estimate

$$
\begin{gathered}
\frac{h}{2} \sum_{j=0}^{N}\left[\left(v_{j}^{\prime}(\tau)-q_{j}^{\prime}(\tau)\right)^{2}+\left(\frac{v_{j+1}(\tau)-v_{j}(\tau)}{h}-\frac{q_{j+1}(\tau)-q_{j}(\tau)}{h}\right)^{2}\right]+\frac{\nu h^{2}}{2}\left(v_{N+1}^{\prime}(\tau)-q_{N+1}^{\prime}(\tau)\right)^{2} \\
\leqslant \beta\left(\frac{h}{2} \sum_{j=0}^{N}\left[\left(v_{j}^{1}-q_{j}^{1}\right)^{2}+\left(\frac{v_{j+1}^{0}-v_{j}^{0}}{h}-\frac{q_{j+1}^{0}-q_{j}^{0}}{h}\right)^{2}\right]+\frac{\nu h^{2}}{2}\left(v_{N+1}^{1}-q_{N+1}^{1}\right)^{2}\right) \\
+C \int_{0}^{\tau}\left[\left(v_{N+1}^{\prime}-y\right)^{2}+h^{2}\left(v_{N+1}^{\prime \prime}-y^{\prime}\right)^{2}\right] d s
\end{gathered}
$$

Let us define $w_{b, h}:=v_{b, h}-q_{b, h}$, where $v_{b, h}$ and $q_{b, h}$ are the solutions of (47) and (14), respectively. We can check that $w_{b, h}=\left(w_{b, j}\right)_{0 \leqslant j \leqslant N+1}$ satisfies

$$
\begin{cases}w_{b, j}^{\prime \prime}-\frac{w_{b, j+1}-2 w_{b, j}+w_{b, j-1}}{h^{2}}+\nu\left(w_{b, j+1}^{\prime}-2 w_{b, j}^{\prime}+w_{b, j-1}^{\prime}\right)=0 & \text { in }(0, \tau), 1 \leqslant j \leqslant N \\ w_{b, 0}=0 & \text { in }(0, \tau), \\ \frac{w_{b, N+1}-w_{b, N}}{h}-w_{b, N+1}^{\prime}=-\left(v_{b, N+1}^{\prime}-y\right) & \text { in }(0, \tau), \\ w_{b, j}(\tau)=v_{b, j}(\tau)-q_{b, j}^{0}, \quad w_{b, j}^{\prime}(\tau)=v_{b, j}^{\prime}(\tau)-q_{b, j}^{1} & 0 \leqslant j \leqslant N+1 .\end{cases}
$$

Thus, $w_{h}(t):=w_{b, h}(\tau-t, x)$ satisfies $(29)$ with $w_{j}^{0}:=v_{b, j}(\tau)-q_{b, j}^{0}$ and $w_{j}^{1}:=-v_{b, j}^{\prime}(\tau)+q_{b, j}^{1}$, for $0 \leq j \leqslant N+1$, and with

$$
\eta(t):=-\left(v_{b, N+1}^{\prime}-y\right)(\tau-t)
$$


Using Lemma 3.2, we obtain

$$
\begin{gathered}
\frac{h}{2} \sum_{j=0}^{N}\left[\left(v_{b, j}^{\prime}(0)-q_{b, j}^{\prime}(0)\right)^{2}+\left(\frac{v_{b, j+1}(0)-v_{b, j}(0)}{h}-\frac{q_{b, j+1}(0)-q_{b, j}(0)}{h}\right)^{2}\right] \\
+\frac{\nu h^{2}}{2}\left(v_{b, N+1}^{\prime}(0)-q_{b, N+1}^{\prime}(0)\right)^{2} \\
\leqslant \beta\left(\frac { h } { 2 } \sum _ { j = 0 } ^ { N } \left[\begin{array}{r}
\left.\left(v_{b, j}^{\prime}(\tau)-q_{b, j}^{1}\right)^{2}+\left(\frac{v_{b, j+1}(\tau)-v_{b, j}(\tau)}{h}-\frac{q_{b, j+1}^{0}-q_{b, j}^{0}}{h}\right)^{2}\right] \\
\left.+\frac{\nu h^{2}}{2}\left(v_{b, N+1}^{\prime}(\tau)-q_{b, N+1}^{1}\right)^{2}\right) \\
+C \int_{0}^{\tau}\left[\left(v_{b, N+1}^{\prime}-y\right)^{2}+h^{2}\left(v_{b, N+1}^{\prime \prime}-y^{\prime}\right)^{2}\right] d s
\end{array}\right.\right.
\end{gathered}
$$

Notice that for $\left(q_{b, h}^{0}, q_{b, h}^{1}\right)=\left(q_{h}(\tau), q_{h}^{\prime}(\tau)\right)=F_{y, h}\left(q_{h}^{0}, q_{h}^{1}\right)$ and taking

$$
v_{b, h}(\tau):=v_{h}(\tau) \quad v_{b, h}^{\prime}(\tau):=v_{h}^{\prime}(\tau)
$$

in (47), we get from (69) and (70) that

$$
\begin{gathered}
\frac{h}{2} \sum_{j=0}^{N}\left[\left(v_{b, j}^{\prime}(0)-q_{b, j}^{\prime}(0)\right)^{2}+\right. \\
\left.\left.+\frac{v_{b, j+1}(0)-v_{b, j}(0)}{h}-\frac{q_{b, j+1}(0)-q_{b, j}(0)}{h}\right)^{2}\right] \\
\quad \frac{\nu h^{2}}{2}\left(v_{b, N+1}^{\prime}(0)-q_{b, N+1}^{\prime}(0)\right)^{2} \\
\leqslant \beta^{2}\left(\frac{h}{2} \sum_{j=0}^{N}\left[\left(v_{j}^{1}-q_{j}^{1}\right)^{2}+\left(\frac{v_{j+1}^{0}-v_{j}^{0}}{h}-\frac{q_{j+1}^{0}-q_{j}^{0}}{h}\right)^{2}\right]+\frac{\nu h^{2}}{2}\left(v_{N+1}^{1}-q_{N+1}^{1}\right)^{2}\right) \\
+C \int_{0}^{\tau}\left[\left(v_{N+1}^{\prime}-y\right)^{2}+h^{2}\left(v_{N+1}^{\prime \prime}-y^{\prime}\right)^{2}\right] d s \\
+C \int_{0}^{\tau}\left[\left(v_{b, N+1}^{\prime}-y\right)^{2}+h^{2}\left(v_{b, N+1}^{\prime \prime}-y^{\prime}\right)^{2}\right] d s .
\end{gathered}
$$

From the above estimate and using that for $\theta \geqslant 0$

$$
(a+b)^{2} \leqslant(1+\theta) a^{2}+\left(1+\frac{1}{\theta}\right) b^{2},
$$


we deduce

$$
\begin{gathered}
\frac{h}{2} \sum_{j=0}^{N}\left[\left(v_{j}^{1}-q_{b, j}^{\prime}(0)\right)^{2}+\left(\frac{v_{j+1}^{0}-v_{j}^{0}}{h}-\frac{q_{b, j+1}(0)-q_{b, j}(0)}{h}\right)^{2}\right]+\frac{\nu h^{2}}{2}\left(v_{N+1}^{1}-q_{b, N+1}^{\prime}(0)\right)^{2} \\
\leqslant\left(1+\frac{1}{\theta}\right)\left(\frac{h}{2} \sum_{j=0}^{N}\left[\left(v_{j}^{1}-v_{b, j}^{\prime}(0)\right)^{2}+\left(\frac{v_{j+1}^{0}-v_{j}^{0}}{h}-\frac{v_{b, j+1}(0)-v_{b, j}(0)}{h}\right)^{2}\right]\right. \\
\left.\quad+\frac{\nu h^{2}}{2}\left(v_{N+1}^{1}-v_{b, N+1}^{\prime}(0)\right)^{2}\right) \\
+(1+\theta) \beta^{2}\left(\frac{h}{2} \sum_{j=0}^{N}\left[\left(v_{j}^{1}-q_{j}^{1}\right)^{2}+\left(\frac{v_{j+1}^{0}-v_{j}^{0}}{h}-\frac{q_{j+1}^{0}-q_{j}^{0}}{h}\right)^{2}\right]+\frac{\nu h^{2}}{2}\left(v_{N+1}^{1}-q_{N+1}^{1}\right)^{2}\right) \\
+C \int_{0}^{\tau}\left[\left(v_{N+1}^{\prime}-y\right)^{2}+h^{2}\left(v_{N+1}^{\prime \prime}-y^{\prime}\right)^{2}\right] d s \\
+C \int_{0}^{\tau}\left[\left(v_{b, N+1}^{\prime}-y\right)^{2}+h^{2}\left(v_{b, N+1}^{\prime \prime}-y^{\prime}\right)^{2}\right] d s
\end{gathered}
$$

with $\theta$ small enough so that

$$
\gamma:=(1+\theta)^{1 / 2} \beta \in(0,1)
$$

Let us set

$$
\begin{gathered}
\varepsilon_{h}=\left(1+\frac{1}{\theta}\right)\left(\frac{h}{2} \sum_{j=0}^{N}\left[\left(v_{j}^{1}-v_{b, j}^{\prime}(0)\right)^{2}+\left(\frac{v_{j+1}^{0}-v_{j}^{0}}{h}-\frac{v_{b, j+1}(0)-v_{b, j}(0)}{h}\right)^{2}\right]\right. \\
\left.+\frac{\nu h^{2}}{2}\left(v_{N+1}^{1}-v_{b, N+1}^{\prime}(0)\right)^{2}\right) \\
+C \int_{0}^{\tau}\left[\left(v_{N+1}^{\prime}-y\right)^{2}+h^{2}\left(v_{N+1}^{\prime \prime}-y^{\prime}\right)^{2}\right] d s \\
+C \int_{0}^{\tau}\left[\left(v_{b, N+1}^{\prime}-y\right)^{2}+h^{2}\left(v_{b, N+1}^{\prime \prime}-y^{\prime}\right)^{2}\right] d s .
\end{gathered}
$$

Note that $\varepsilon_{h}$ does not depend on $\left(q_{h}^{0}, q_{h}^{1}\right)$. Using (51), (55), (56), (57), (60) and (61) we obtain

$$
\varepsilon_{h} \rightarrow 0 \quad \text { as } h \rightarrow 0 .
$$

From (72) and (73), we deduce that

$$
\begin{aligned}
E_{h}\left(\left(v_{h}^{0}-q_{b, h}(0)\right),\left(v_{h}^{1}-q_{b, h}^{\prime}(0)\right)\right) & +\frac{\nu h^{2}}{2}\left(v_{N+1}^{1}-q_{b, N+1}^{\prime}(0)\right)^{2} \\
& \leqslant \varepsilon_{h}+\gamma^{2}\left(E_{h}\left(\left(v_{h}^{0}-q_{h}^{0}\right),\left(v_{h}^{1}-q_{h}^{1}\right)\right)+\frac{\nu h^{2}}{2}\left(v_{N+1}^{1}-q_{N+1}^{1}\right)^{2}\right) .
\end{aligned}
$$

Consequently, we can proceed by induction, considering that $\left(q_{b, h}(0), q_{b, h}^{\prime}(0)\right)=B_{y, h} \circ F_{y, h}\left(q_{h}^{0}, q_{h}^{1}\right)$, we deduce the result. 


\section{Numerical illustrations}

This section is devoted to some numerical illustrations of the method proposed in this paper.

The numerical scheme that we use is slightly different from (12), (14). We replace them by

$$
\begin{cases}q_{j}^{\prime \prime}-\frac{q_{j+1}-2 q_{j}+q_{j-1}}{h^{2}}-\nu\left(q_{j+1}^{\prime}-2 q_{j}^{\prime}+q_{j-1}^{\prime}\right)=0 & \text { in }(0, \tau), 1 \leqslant j \leqslant N, \\ q_{0}=0 & \text { in }(0, \tau), \\ \frac{q_{N+1}-q_{N}}{h}+\mu q_{N+1}^{\prime}=\mu y & \text { in }(0, \tau), \\ q_{j}(0)=q_{j}^{0}, \quad q_{j}^{\prime}(0)=q_{j}^{1} & \text { for } j=0, \cdots, N+1,\end{cases}
$$

and

$$
\begin{cases}q_{b, j}^{\prime \prime}-\frac{q_{b, j+1}-2 q_{b, j}+q_{b, j-1}}{h^{2}}+\nu\left(q_{b, j+1}^{\prime}-2 q_{b, j}^{\prime}+q_{b, j-1}^{\prime}\right)=0 & \text { in }(0, \tau), 1 \leqslant j \leqslant N, \\ q_{b, 0}=0 & \text { in }(0, \tau), \\ \frac{q_{b, N+1}-q_{b, N}}{h}-\mu q_{b, N+1}^{\prime}=-\mu y & \text { in }(0, \tau), \\ q_{b, j}(\tau)=q_{b, j}^{0}, \quad q_{b, j}^{\prime}(\tau)=q_{b, j}^{1} & \text { for } j=0, \cdots, N+1,\end{cases}
$$

where $\mu$ is a positive constant that is chosen to be 1 in (12), (14). The reason for this change is that all the results we proved are still valid in that case, but for $\mu=1$, we may have a better result. Indeed, for system (16), one can prove that for $\tau>2, w(\tau)=\partial_{t} w(\tau)=0$. This means that in particular in Proposition 1.1, we only need one iteration $(n=1)$ to recover exactly $v^{0}$ and $v^{1}$. For the numerical observers, based on this result, we can hope that in this particular example, we may not need a numerical viscosity in this particular example and with $\mu=1$. Nevertheless, this property of equation (1) is quite particular and our aim is to precise this example in order to understand how we can use numerical viscosity in such a method. We developed some extensions in Section 7.

Another important aspect for the numerical simulation is the discretization in time. We have not dealt with this problem here, but this is an important issue that was considered in the context of numerical viscosity in [27], [28], [26], etc. Here we have discretized the second derivatives by using the classical scheme

$$
q_{j}^{\prime \prime}\left(t^{n}\right) \approx \frac{q_{j}^{n+1}-2 q_{j}^{n}+q_{j}^{n-1}}{(\delta t)^{2}}
$$

and for the first derivative in (76), we take

$$
q_{j}^{\prime}\left(t^{n}\right) \approx \frac{q_{j}^{n}-q_{j}^{n-1}}{\delta t}
$$

This leads to an explicit scheme in time and thus we take in the examples below $(\delta t) / h$ small enough (CFL condition).

In our first example, we take

$$
v^{0}(x)=x(1-x)^{2} \sin (15 x), \quad v^{1}(x)=-x \quad(x \in[0,1]) .
$$

We show in Figure 1 several iterations of the iterative method $(n \in\{2,4,6,8,10\})$.

In this test, we take $\mu=5, \nu=3$ and a mesh size $h=1 /(N+1)$ with $N=240$. We also discretized the time interval, with a time step $\delta t=\tau / M$, with $M=1200$, and $\tau=2.5$. We notice that the reconstruction of the velocity is less accurate that the reconstruction of the position. This may come from the discretization of velocity by using(79). 

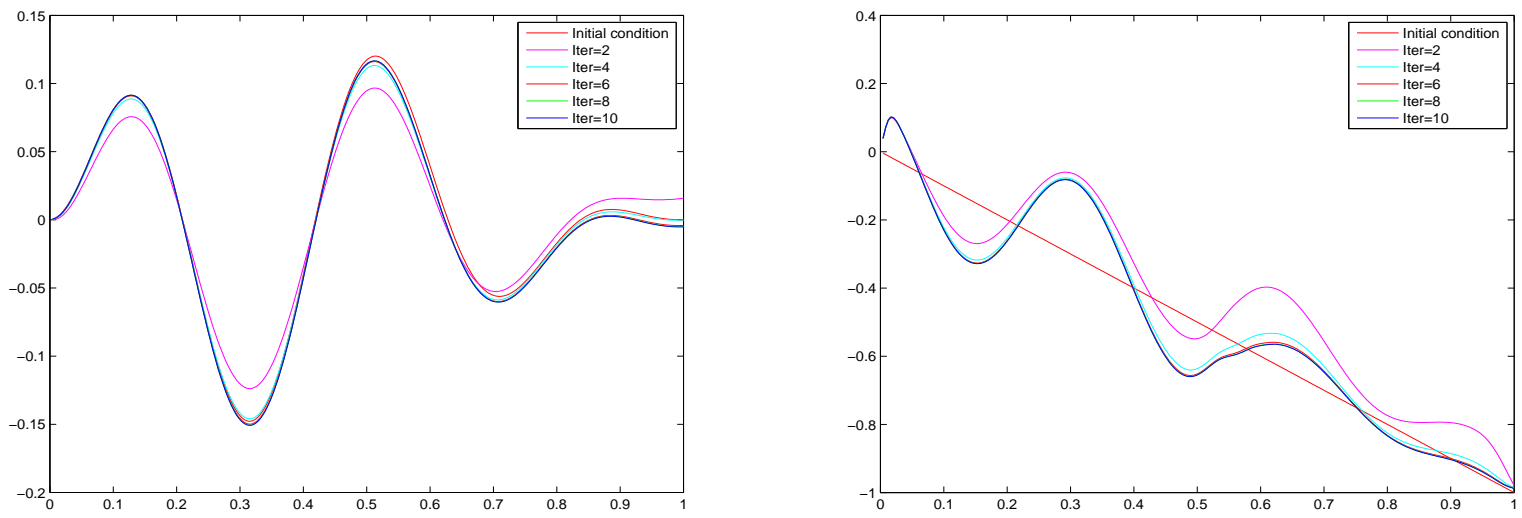

Figure 1: Reconstruction of $v^{0}$ (at the left) and of $v^{1}$ (at the right)

Let us recall the error term considered in our analysis:

$$
E_{h}=\frac{h}{2} \sum_{j=0}^{N}\left[\left(b_{j}^{(n)}-v_{j}^{1}\right)^{2}+\left(\frac{a_{j+1}^{(n)}-a_{j}^{(n)}}{h}-\frac{v_{j+1}^{0}-v_{j}^{0}}{h}\right)^{2}\right]
$$

where $v_{j}^{0}=v^{0}(j h), v_{j}^{1}=v^{1}(j h)$ for $0 \leqslant j \leqslant N+1$ and

$$
\left(a_{h}^{(k)}, b_{h}^{(k)}\right)=\left(B_{y, h} \circ F_{y, h}\right)\left(\left(a_{h}^{(k-1)}, b_{h}^{(k-1)}\right)\right), \quad k \in\{1, \ldots, n\},
$$

where here, we take for initial guess $\left(a_{h}^{(0)}, b_{h}^{(0)}\right)=(0,0)$.

We represent in Figure 2 the relative error made after each iteration.

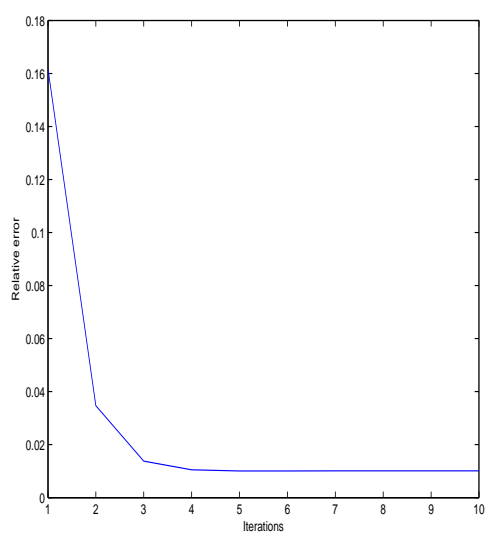

Figure 2: Relative error made after $n$ iterations

We can see the influence of the viscosity $\nu$ : let us take

$$
v^{0}(x)=\sin \left(\frac{31 \pi x}{2}\right), \quad v^{1}(x)=-x \quad(x \in[0,1]) .
$$

For instance in Figure 3, we show the relative error made for $\mu=5, N=240, M=1200, \tau=2.5$ and $n=10$. 


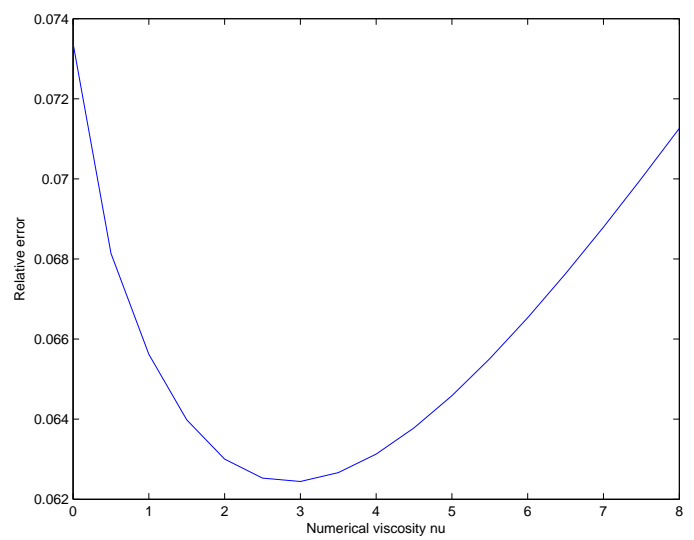

Figure 3: Relative error with respect to $\nu$

This illustrates the fact that the factor of the vanishing viscosity need to be chosen carefully. Here we see that there is an optimal choice of $\nu_{*} \approx 3$. Such an optimal factor may depend on the other parameters of the problem.

We also show how the the time $\tau$ plays a role in the method. We take

$$
v^{0}(x)=x \sin \left(\frac{31 \pi x}{2}\right), \quad v^{1}(x)=-x \quad(x \in[0,1])
$$

and $\mu=5, N=240, M=1200, \nu=3$ and $n=10$. We represent in Figure 4 the final reconstruction of the initial condition for the position and the relative error for $\tau \in\{1.5,1.8,2.1,2.5\}$.
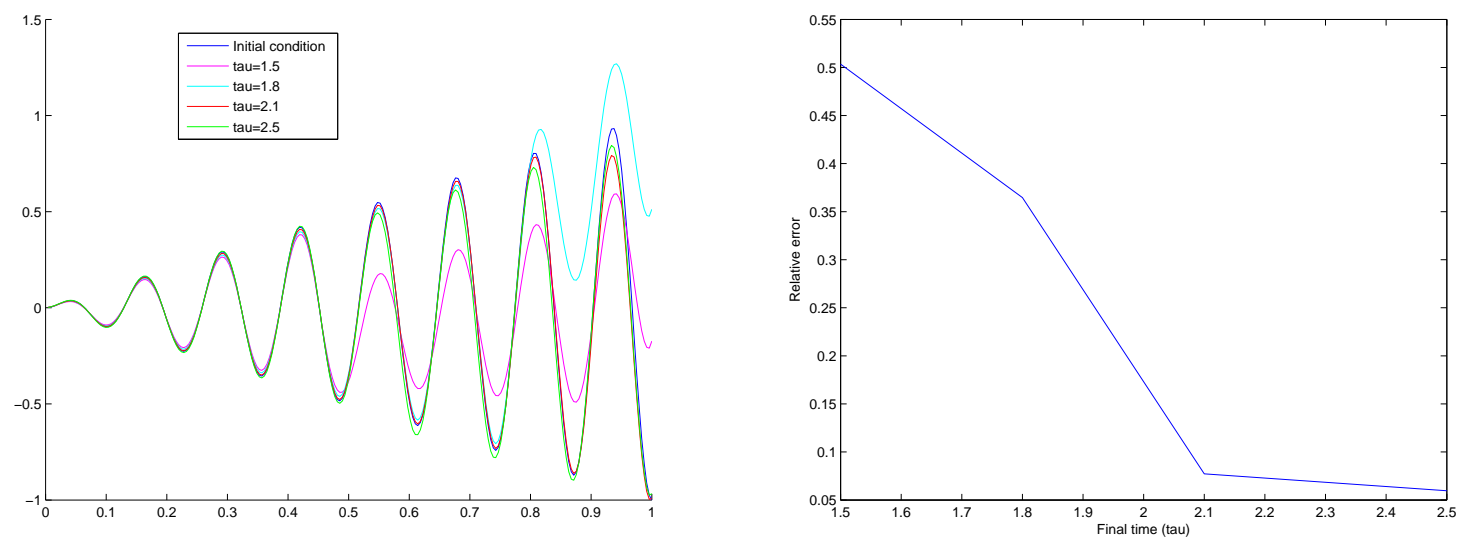

Figure 4: Reconstruction of $v^{0}$ and relative error with respect to $\tau$

In particular, we notice that if $\tau<2$, then the method does not give a good approximation of the initial conditions. In [13] and [14], the author tackles the problem of reconstructing the initial conditions if we lose the exact observability (as for $\tau<2$ ) and shows that a set of initial conditions can still be reconstructed.

Even if our result (Theorem 1.2) needs regular initial conditions, we can use our method to try to reconstruct initial conditions with less regularity than in the main result. For instance, we take

$$
v^{0}(x)=x, \quad v^{1}(x)=-1_{(0,1 / 2)}(x) .
$$


We still have $v^{0} \in H_{L}^{1}(0,1)$ and $v^{1} \in L^{2}(0,1)$ in that case. We obtain in that case, with $\mu=5, \tau=2.5$, $N=240, M=1200, \nu=3$ and $n=10$ the figure 5 where we represent the reconstruction of the initial conditions and the relative error. We notice that in that case, the algorithm is still converging
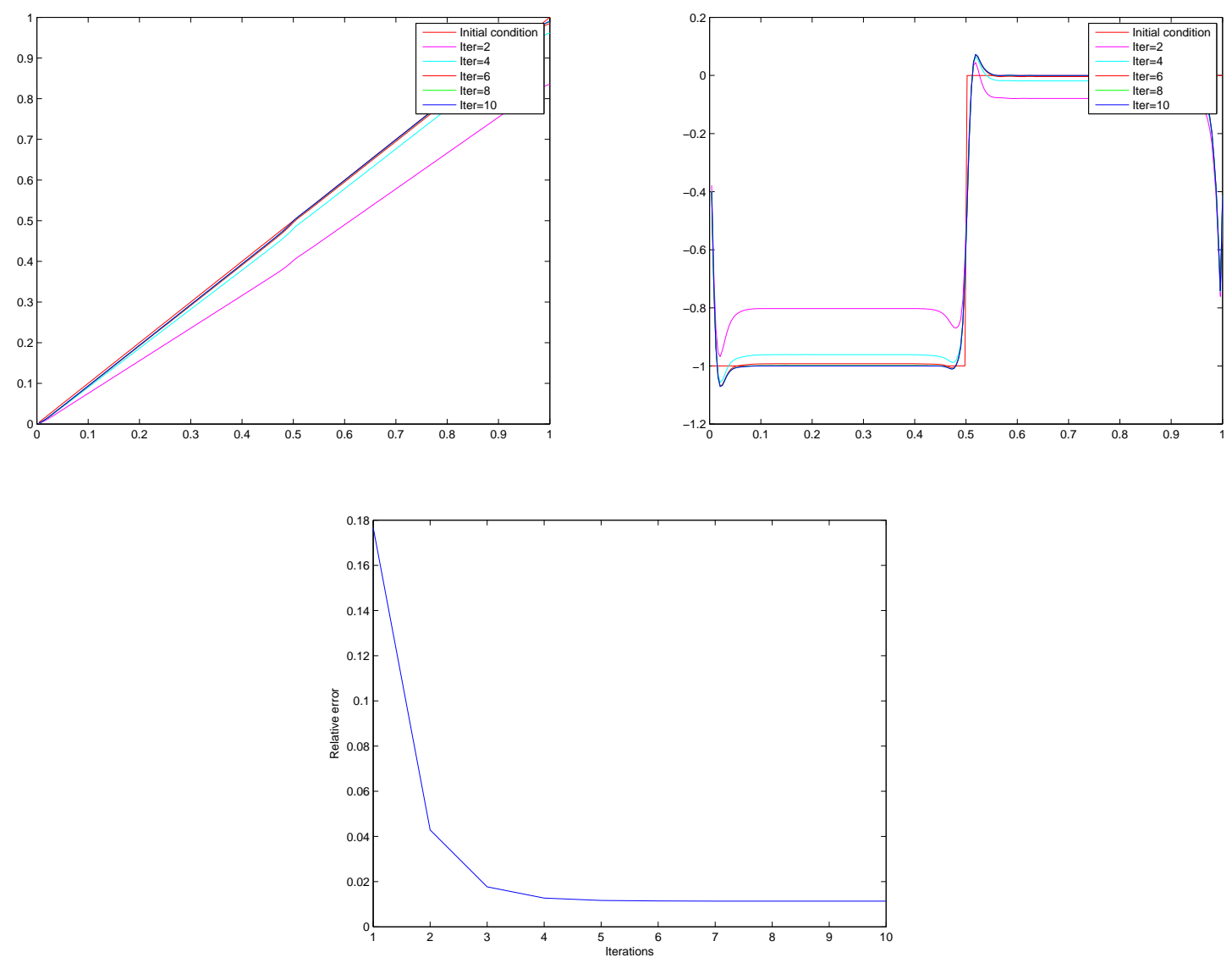

Figure 5: Reconstruction of $v^{0}, v^{1}$ and relative error made after $n$ iterations

and allows to reconstruct such initial conditions.

Finally, we present a last experiment where we add in the observation $y$ a random perturbation. More precisely, instead of giving $y\left(t^{k}\right)$ to the numerical scheme, we give $y\left(t^{k}\right)+X^{k}$, where $\left(X^{k}\right)_{k}$ is a random vector, normally distributed with mean 0 and deviation $\sigma$.

We take $\mu=5, \tau=2.5, N=240, M=1200, \nu=3$ and $n=10$ and

$$
v^{0}(x)=x(1-x)^{2} \sin (15 x), \quad v^{1}(x)=-x \quad(x \in[0,1])
$$

Figure 6 represents the mean of ten experiments with independent perturbations.

\section{Remarks on the vanishing viscosity for numerical observers}

In this paper, we focus on the numerical counterpart of the method developed in [34] for recovering initial conditions by using observers. More precisely, we detailed a way to discretize their method for the $1 \mathrm{~d}$ wave equation. However, this approach may be applied to many different problems. In this section, we present some possible generalizations. 

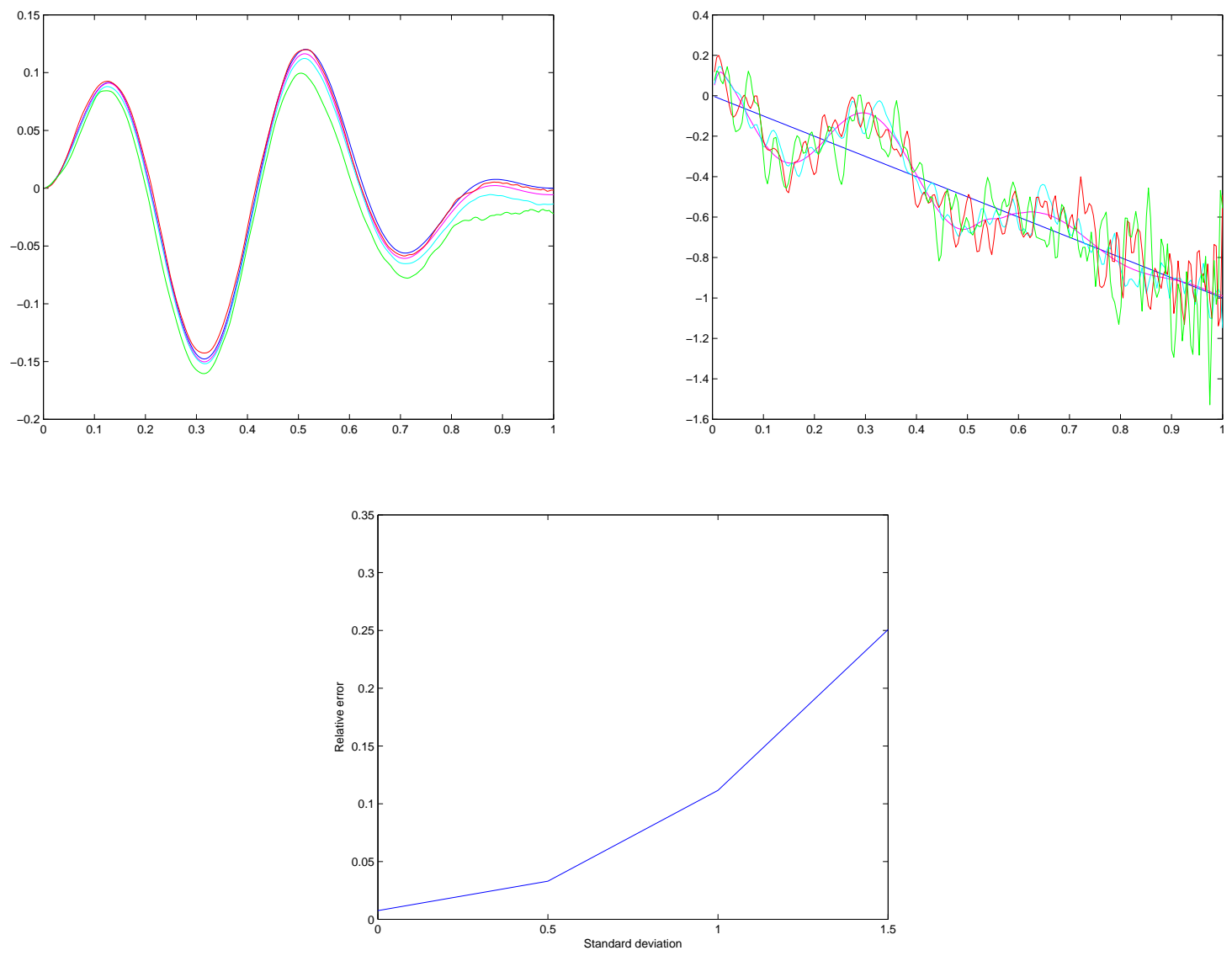

Figure 6: Reconstruction of $v^{0}, v^{1}$ and relative error with random perturbation

First let us consider a general class of hyperbolic systems of second order: we introduce $H$ and $U$ two real Hilbert spaces, $A_{0}: D\left(A_{0}\right) \rightarrow H$ a self-adjoint, positive operator and $C_{0} \in \mathcal{L}(H, U)$ an observation admissible operator. We consider the system

$$
\begin{gathered}
\ddot{v}+A_{0} v=0 \quad \text { in }(0, \tau), \\
v(0)=v^{0}, \quad \dot{v}(0)=v^{1}, \\
y=C_{0} \dot{v} \quad \text { in }(0, \tau) .
\end{gathered}
$$

Let us make the hypothesis that

$$
\begin{gathered}
\ddot{w}+A_{0} w+C_{0}^{*} C_{0} \dot{w}=0 \quad \text { in }(0, \tau), \\
w(0)=w^{0}, \dot{w}(0)=w^{1},
\end{gathered}
$$

is exponentially stable:

$$
\left(\|\dot{w}(t)\|_{H}+\left\|A_{0}^{1 / 2} w(t)\right\|_{H}\right) \leqslant M_{0} e^{-\alpha_{0} t}\left(\left\|w^{1}\right\|_{H}+\left\|A_{0}^{1 / 2} w^{0}\right\|_{H}\right) \quad \forall\left(w^{0}, w^{1}\right) \in D\left(A_{0}^{1 / 2}\right) \times H .
$$

Such an hypothesis is sufficient to obtain the convergence of the algorithm of [34]. Indeed they assume $C_{0} \in \mathcal{L}\left(D\left(A_{0}^{1 / 2}\right), U\right)$ is an observation admissible operator (not necessary bounded) but we choose to write this abstract generalization only in the case of a bounded observation operator. 
Let us present how to recover approximations $v_{h}^{0}$ and $v_{h}^{1}$ of $v^{0}$ and $v^{1}$ by using an approximation of $y$ with a method of numerical observers.

Assume that $A_{0 h} \in \mathcal{L}\left(V_{h}\right)$ (resp. $\quad C_{0 h} \in \mathcal{L}\left(V_{h}, U_{h}\right)$ ) are finite dimensional approximations of $A_{0}$ (resp. $C_{0}$ ) obtained by using a classical method of discretization (finite element method, finite difference method, etc.). Let us consider $\nu \geqslant 0$ and $\theta>0$ some real numbers and $y_{h} \in U_{h}$ an approximation of $y$. Then the numerical observers of (81)-(83) can be written as

$$
F_{h}: V_{h} \times V_{h} \rightarrow V_{h} \times V_{h}, \quad\left(q_{h}^{0}, q_{h}^{1}\right) \mapsto\left(q_{h}(\tau), \dot{q}_{h}(\tau)\right)
$$

and

$$
B_{h}: V_{h} \times V_{h} \rightarrow V_{h} \times V_{h}, \quad\left(q_{b, h}^{0}, q_{b, h}^{1}\right) \mapsto\left(q_{b, h}(0), \dot{q}_{b, h}(0)\right)
$$

where

$$
\begin{gathered}
\ddot{q}_{h}+A_{0 h} q_{h}+C_{0 h}^{*} C_{0 h} \dot{q}_{h}-C_{0 h}^{*} y_{h}+\nu h^{\theta} A_{0 h} \dot{q}_{h}=0 \quad \text { in }(0, \tau), \\
q_{h}(0)=q_{h}^{0}, \dot{q}_{h}(0)=q_{h}^{1},
\end{gathered}
$$

and

$$
\begin{gathered}
\ddot{q}_{b, h}+A_{0 h} q_{b, h}-C_{0 h}^{*} C_{0 h} \dot{q}_{b, h}+C_{0 h}^{*} y_{h}-\nu h^{\theta} A_{0 h} \dot{q}_{b, h}=0 \quad \text { in }(0, \tau), \\
q_{b, h}(\tau)=q_{b, h}^{0}, \dot{q}_{b, h}(\tau)=q_{b, h}^{1},
\end{gathered}
$$

We assume that the numerical viscosity terms $\nu h^{\theta} A_{0 h} \dot{q}_{h}$ and $-\nu h^{\theta} A_{0 h} \dot{q}_{b, h}$ are used to obtain the numerical counterpart of (86). More precisely, let us consider the finite-dimensional approximations of the system (84)-(85):

$$
\begin{gathered}
\ddot{w}_{h}+A_{0, h} w_{h}+C_{0 h}^{*} C_{0 h} \dot{w}_{h}+\nu h^{\theta} A_{0 h} \dot{w}_{h}=0 \quad \text { in }(0, \tau), \\
w_{h}(0)=w_{h}^{0}, \dot{w}_{h}(0)=w_{h}^{1} .
\end{gathered}
$$

We assume that $\nu \geqslant 0$ and $\theta>0$ are chosen so that there exist $M>0$ and $\alpha>0$ so that for all $h$,

$$
\left(\left\|\dot{w}_{h}(t)\right\|_{V_{h}}+\left\|A_{0 h}^{1 / 2} w_{h}(t)\right\|_{V_{h}}\right) \leqslant M e^{-\alpha t}\left(\left\|w_{h}^{1}\right\|_{V_{h}}+\left\|A_{0 h}^{1 / 2} w_{h}^{0}\right\|_{V_{h}}\right) \quad \forall\left(w_{h}^{0}, w_{h}^{1}\right) \in V_{h} \times V_{h} .
$$

Remark 7.1. Let us emphasize that without the numerical viscosity $\nu h^{\theta} A_{0 h} \dot{w}_{h}$, the property (95) may be false (see, for instance, [12],[17], [18]). The use of artificial numerical viscosity to ensure the uniform exponential decay (95) was considered in several partial differential equations or classes of partial differential equations. Let us quote some of them that can be used as examples of application of this section:

- 2D wave equation with finite-difference space semi-discretization ([35]);

- 2D plate equation with finite-difference space semi-discretization ([31]);

- a class of second order evolution equations (with $C_{0}$ bounded and the spectrum of $A_{0}$ consisting of isolated eigenvalues with a gap between them) ([33]).

Let us also remark that for the 1D plate equation with finite-difference space semi-discretization, it is proved in [32] that (95) holds without the add of a numerical viscosity $(\nu=0)$.

Let us consider the sequence

$$
\left(a_{h}^{(n)}, b_{h}^{(n)}\right)=\left(B_{h} \circ F_{h}\right)\left(\left(a_{h}^{(n-1)}, b_{h}^{(n-1)}\right)\right), \quad n \in \mathbb{N}^{*},
$$


where $\left(a_{h}^{(0)}, b_{h}^{(0)}\right)$ is an initial guess of $\left(v_{h}^{0}, v_{h}^{1}\right)$.

We also assume the following technical hypothesis: there exists $M>0$ such that for all $h$ small enough

$$
\left\|C_{0 h}^{*}\right\|_{\mathcal{L}\left(U_{h}, V_{h}\right)} \leqslant M .
$$

Finally, we assume the following hypothesis on the numerical schemes with numerical viscosity: let us consider $v_{h}$ and $v_{b, h}$ the solutions of

$$
\begin{gathered}
\ddot{v}_{h}+A_{0 h} v_{h}+\nu h^{\theta} A_{0 h} \dot{v}_{h}=0 \quad \text { in }(0, \tau), \\
v_{h}(0)=v_{h}^{0}, \dot{v}_{h}(0)=v_{h}^{1},
\end{gathered}
$$

and

$$
\begin{gathered}
\ddot{v}_{b, h}+A_{0 h} v_{b, h}-\nu h^{\theta} A_{0 h} \dot{v}_{b, h}=0 \quad \text { in }(0, \tau), \\
v_{b, h}(\tau)=v_{b, h}^{0}, \dot{v}_{b, h}(\tau)=v_{b, h}^{1} . \\
\int_{0}^{\tau}\left\|C_{0 h} \dot{v}_{h}-y_{h}\right\|_{U_{h}} d s+\int_{0}^{\tau}\left\|C_{0 h} \dot{v}_{b, h}-y_{h}\right\|_{U_{h}} d s \rightarrow 0
\end{gathered}
$$

and if $v_{h}(\tau)=v_{b, h}(\tau), \dot{v}_{h}(\tau)=\dot{v}_{b, h}(\tau)$, then

$$
\left\|\dot{v}_{b, h}(0)-v_{h}^{1}\right\|_{V_{h}}+\left\|A_{0 h}^{1 / 2}\left(v_{b, h}(0)-v_{h}^{0}\right)\right\|_{V_{h}} \rightarrow 0 .
$$

Note that the above condition always holds true if $\nu=0$. Recall that $v_{h}^{0}$ and $v_{h}^{1}$ are approximations of $v^{0}$ and $v^{1}$.

Theorem 7.2. Assume the above hypotheses. Then there exist $\tau>0, \beta \in(0,1)$ and $\varepsilon_{h}>0$ such that for all $n$,

$$
\left\|b_{h}^{n}-v_{h}^{1}\right\|_{V_{h}}+\left\|A_{0 h}^{1 / 2}\left(a_{h}^{n}-v_{h}^{0}\right)\right\|_{V_{h}} \leqslant \beta^{2 n}\left(\left\|b_{h}^{0}-v_{h}^{1}\right\|_{V_{h}}+\left\|A_{0 h}^{1 / 2}\left(a_{h}^{0}-v_{h}^{0}\right)\right\|_{V_{h}}\right)+\frac{1}{1-\beta^{2}} \varepsilon_{h},
$$

where $\varepsilon_{h}$ is independent of $n$ and goes to 0 as $h \rightarrow 0$.

Remark 7.3. Let us emphasize that in this abstract result, the time $\tau$ used to construct the numerical observers may be quite large and is obtained in the proof by using (95). The minimal time $\tau$ should be related, as in the case of the $1 \mathrm{~d}$ wave equation, to the observability inequality.

Proof of Theorem 7.2. We notice that $w_{h}:=v_{h}-q_{h}$ satisfies

$$
\begin{gathered}
\ddot{w}_{h}+A_{0 h} w_{h}+C_{0 h}^{*} C_{0 h} \dot{w}_{h}+\nu h^{\theta} A_{0 h} \dot{w}_{h}=C_{0 h}^{*} \eta_{h} \quad \text { in }(0, \tau), \\
w_{h}(0)=v_{h}^{0}-q_{h}^{0}, \dot{w}_{h}(0)=v_{h}^{1}-q_{h}^{1},
\end{gathered}
$$

with

$$
\eta_{h}:=C_{0 h} \dot{v}_{h}-y_{h}
$$

We can write (103)-(104) as

$$
\frac{d}{d t}\left[\begin{array}{c}
w_{h} \\
\dot{w}_{h}
\end{array}\right]=\left[\begin{array}{cc}
0 & I \\
-A_{0 h} & -C_{0 h}^{*} C_{0 h}-\nu h^{\theta} A_{0 h}
\end{array}\right]\left[\begin{array}{c}
w_{h} \\
\dot{w}_{h}
\end{array}\right]+\left[\begin{array}{c}
0 \\
C_{0 h}^{*} \eta_{h}
\end{array}\right]
$$

and using (95), we deduce that there exist $\tau>0$ and $\beta<1$ such that for all $t \geqslant \tau$,

$$
\left(\left\|\dot{w}_{h}(t)\right\|_{V_{h}}+\left\|A_{0 h}^{1 / 2} w_{h}(t)\right\|_{V_{h}}\right) \leqslant \beta\left(\left\|v_{h}^{1}-q_{h}^{1}\right\|_{V_{h}}+\left\|A_{0 h}^{1 / 2}\left(v_{h}^{0}-q_{h}^{0}\right)\right\|_{V_{h}}\right)+\int_{0}^{t}\left\|C_{0 h}^{*} \eta_{h}\right\|_{V_{h}} d s .
$$


In particular,

$$
\begin{array}{r}
\left(\left\|\dot{v}_{h}(\tau)-\dot{q}_{h}(\tau)\right\|_{V_{h}}+\left\|A_{0 h}^{1 / 2}\left(v_{h}(\tau)-q_{h}(\tau)\right)\right\|_{V_{h}}\right) \leqslant \beta\left(\left\|v_{h}^{1}-q_{h}^{1}\right\|_{V_{h}}+\left\|A_{0 h}^{1 / 2}\left(v_{h}^{0}-q_{h}^{0}\right)\right\|_{V_{h}}\right) \\
+M \int_{0}^{\tau}\left\|\eta_{h}\right\|_{V_{h}} d s .
\end{array}
$$

In a similar way,

$$
\begin{aligned}
& \left(\left\|\dot{v}_{b, h}(0)-\dot{q}_{b, h}(0)\right\|_{V_{h}}+\left\|A_{0 h}^{1 / 2}\left(v_{b, h}(0)-q_{b, h}(0)\right)\right\|_{V_{h}}\right) \\
& \quad \leqslant \beta\left(\left\|\dot{v}_{b, h}(\tau)-\dot{q}_{b, h}(\tau)\right\|_{V_{h}}+\left\|A_{0 h}^{1 / 2}\left(v_{b, h}(\tau)-q_{b, h}(\tau)\right)\right\|_{V_{h}}\right)+M \int_{0}^{\tau}\left\|\tilde{\eta}_{h}\right\|_{V_{h}} d s
\end{aligned}
$$

where

$$
\tilde{\eta}_{h}:=C_{0 h} \dot{v}_{b, h}-y_{h}
$$

Taking

$$
\begin{array}{ll}
v_{b, h}(\tau)=v_{h}(\tau), & \dot{v}_{b, h}(\tau)=\dot{v}_{h}(\tau) \\
q_{b, h}(\tau)=q_{h}(\tau), & \dot{q}_{b, h}(\tau)=\dot{q}_{h}(\tau)
\end{array}
$$

we deduce from (108) and from (109) that

$$
\begin{aligned}
& \left(\left\|\dot{v}_{b, h}(0)-\dot{q}_{b, h}(0)\right\|_{V_{h}}+\left\|A_{0 h}^{1 / 2}\left(v_{b, h}(0)-q_{b, h}(0)\right)\right\|_{V_{h}}\right) \\
& \quad \leqslant \beta^{2}\left(\left\|v_{h}^{1}-q_{h}^{1}\right\|_{V_{h}}+\left\|A_{0 h}^{1 / 2}\left(v_{h}^{0}-q_{h}^{0}\right)\right\|_{V_{h}}\right)+M \int_{0}^{\tau}\left\|\eta_{h}\right\|_{V_{h}} d s+M \int_{0}^{\tau}\left\|\tilde{\eta}_{h}\right\|_{V_{h}} d s .
\end{aligned}
$$

The above estimate yields

$$
\begin{aligned}
& \left(\left\|v_{h}^{1}-\dot{q}_{b, h}(0)\right\|_{V_{h}}+\left\|A_{0 h}^{1 / 2}\left(v_{h}^{0}-q_{b, h}(0)\right)\right\|_{V_{h}}\right) \\
& \leqslant \beta^{2}\left(\left\|v_{h}^{1}-q_{h}^{1}\right\|_{V_{h}}+\left\|A_{0 h}^{1 / 2}\left(v_{h}^{0}-q_{h}^{0}\right)\right\|_{V_{h}}\right)+M \int_{0}^{\tau}\left\|\eta_{h}\right\|_{V_{h}} d s+M \int_{0}^{\tau}\left\|\tilde{\eta}_{h}\right\|_{V_{h}} d s \\
& +\left\|\dot{v}_{b, h}(0)-v_{h}^{1}\right\|_{V_{h}}+\left\|A_{0 h}^{1 / 2}\left(v_{b, h}(0)-v_{h}^{0}\right)\right\|_{V_{h}} .
\end{aligned}
$$

Using (101) and (102) and proceeding by induction, we deduce the result.

\section{Acknowledgments}

The first author's work was partially supported by Fondecyt Conicyt 1120560 project and CONICYT Anillo ACPA ACT1106 grants. The second author's work was partially supported by Fondecyt Conicyt 1120560 project and by ANR Grant CISIFS (09-BLAN-0213-02).

\section{References}

[1] Didier Auroux and Jacques Blum. Back and forth nudging algorithm for data assimilation problems. C. R. Math. Acad. Sci. Paris, 340(12):873-878, 2005.

[2] H. T. Banks, K. Ito, and C. Wang. Exponentially stable approximations of weakly damped wave equations. In Estimation and control of distributed parameter systems (Vorau, 1990), volume 100 of Internat. Ser. Numer. Math., pages 1-33. Birkhäuser, Basel, 1991. 
[3] Claude Bardos, Gilles Lebeau, and Jeffrey Rauch. Sharp sufficient conditions for the observation, control, and stabilization of waves from the boundary. SIAM J. Control Optim., 30(5):1024-1065, 1992.

[4] A. Bensoussan. Filtrage optimal des systèmes linéaires. Dunod, 1971.

[5] Haïm Brezis. Analyse fonctionnelle. Collection Mathématiques Appliquées pour la Maîtrise. [Collection of Applied Mathematics for the Master's Degree]. Masson, Paris, 1983. Théorie et applications. [Theory and applications].

[6] D. Chapelle, N. Cîndea, and P. Moireau. Improving convergence in numerical analysis using observers - the wave-like equation case. Math. Models Methods Appl. Sci., 22(12):1250040, 35, 2012.

[7] Dominique Chapelle, Nicolae Cîndea, Maya De Buhan, and Philippe Moireau. Exponential convergence of an observer based on partial field measurements for the wave equation. Math. Probl. Eng., pages Art. ID 581053, 12, 2012.

[8] Nicolae Cîndea, Sorin Micu, and Marius Tucsnak. An approximation method for exact controls of vibrating systems. SIAM J. Control Optim., 49(3):1283-1305, 2011.

[9] Ruth F. Curtain and Hans Zwart. An introduction to infinite-dimensional linear systems theory, volume 21 of Texts in Applied Mathematics. Springer-Verlag, New York, 1995.

[10] J. Deguenon, G. Sallet, and C.-Z. Xu. Infinite dimensional observers for vibrating systems. In Proc. of IEEE Conf. on Decision and Control, pages 3979-3983, 2006.

[11] Roland Glowinski, Chin Hsien Li, and Jacques-Louis Lions. A numerical approach to the exact boundary controllability of the wave equation. I. Dirichlet controls: description of the numerical methods. Japan J. Appl. Math., 7(1):1-76, 1990.

[12] Roland Glowinski, Chin Hsien Li, and Jacques-Louis Lions. A numerical approach to the exact boundary controllability of the wave equation. I. Dirichlet controls: description of the numerical methods. Japan J. Appl. Math., 7(1):1-76, 1990.

[13] Ghislain Haine. Recovering the initial data of an evolution equation. Application to thermoacoustic tomography. Submitted, 2012.

[14] Ghislain Haine. Recovering the observable part of the initial data of an infinite-dimensional linear system. Submitted, 2012.

[15] Ghislain Haine and Karim Ramdani. Observateurs itératifs en horizon fini. application à la reconstruction de données initiales pour des edp d'évolution. Journal Européen des Systèmes Automatisés (JESA), 45(7-8-9-10):715-724, 2011.

[16] Ghislain Haine and Karim Ramdani. Reconstructing initial data using observers: error analysis of the semi-discrete and fully discrete approximations. Numer. Math., 120(2):307-343, 2012.

[17] Juan-Antonio Infante and Enrique Zuazua. Boundary observability for the space-discretizations of the 1-d wave equation. C. R. Acad. Sci. Paris Sér. I Math., 326(6):713-718, 1998.

[18] Juan Antonio Infante and Enrique Zuazua. Boundary observability for the space semidiscretizations of the 1-D wave equation. M2AN Math. Model. Numer. Anal., 33(2):407-438, 1999. 
[19] Kazufumi Ito, Karim Ramdani, and Marius Tucsnak. A time reversal based algorithm for solving initial data inverse problems. Discrete Contin. Dyn. Syst. Ser. S, 4(3):641-652, 2011.

[20] T. Kailath, A.H. Sayed, and H. Babak. Linear Estimation. Prentice Hall, 2000.

[21] V. Komornik. Exact controllability and stabilization. RAM: Research in Applied Mathematics. Masson, Paris, 1994. The multiplier method.

[22] Xiao-Dong Li, Cheng-Zhong Xu, Yue-Jun Peng, and Marius Tucsnak. On the numerical investigation of a Luenberger type observer for infinite-dimensional vibrating systems. In Proceedings of the 17th World Congress The International Federation of Automatic Control, pages 7624-7629, Seoul, Corée, République De, 2008.

[23] J.-L. Lions. Contrôlabilité exacte, perturbations et stabilisation de systèmes distribués. Tome 2, volume 9 of Recherches en Mathématiques Appliquées [Research in Applied Mathematics]. Masson, Paris, 1988. Perturbations. [Perturbations].

[24] Sorin Micu. Uniform boundary controllability of a semidiscrete 1-D wave equation with vanishing viscosity. SIAM J. Control Optim., 47(6):2857-2885, 2008.

[25] Philippe Moireau, Dominique Chapelle, and Patrick Le Tallec. Joint state and parameter estimation for distributed mechanical systems. Comput. Methods Appl. Mech. Engrg., 197(6-8):659-677, 2008 .

[26] Arnaud Münch. A uniformly controllable and implicit scheme for the 1-D wave equation. M2AN Math. Model. Numer. Anal., 39(2):377-418, 2005.

[27] Mihaela Negreanu and Enrique Zuazua. Uniform boundary controllability of a discrete 1-D wave equation. Systems Control Lett., 48(3-4):261-279, 2003. Optimization and control of distributed systems.

[28] Mihaela Negreanu and Enrique Zuazua. Discrete Ingham inequalities and applications. SIAM J. Numer. Anal., 44(1):412-448 (electronic), 2006.

[29] Serge Nicaise and Julie Valein. Quasi exponential decay of a finite difference space discretization of the 1-d wave equation by pointwise interior stabilization. Adv. Comput. Math., 32(3):303-334, 2010 .

[30] Kim Dang Phung and Xu Zhang. Time reversal focusing of the initial state for Kirchhoff plate. SIAM J. Appl. Math., 68(6):1535-1556, 2008.

[31] K. Ramdani, T. Takahashi, and M. Tucsnak. Internal stabilization of the plate equation in a square: the continuous and the semi-discretized problems. J. Math. Pures Appl. (9), 85(1):17-37, 2006.

[32] Karim Ramdani, Takéo Takahashi, and Marius Tucsnak. Semi-discrétisation en espace du problème de la stabilisation interne de l'équation des poutres. In Paris-Sud Working Group on Modelling and Scientific Computing 2006-2007, volume 18 of ESAIM Proc., pages 48-56. EDP Sci., Les Ulis, 2007.

[33] Karim Ramdani, Takéo Takahashi, and Marius Tucsnak. Uniformly exponentially stable approximations for a class of second order evolution equations - application to LQR problems. ESAIM Control Optim. Calc. Var., 13(3):503-527, 2007. 
[34] Karim Ramdani, Marius Tucsnak, and George Weiss. Recovering the initial state of an infinitedimensional system using observers. Automatica, 46:1616-1625, 2010.

[35] Louis Roder Tcheugoué Tébou and Enrique Zuazua. Uniform exponential long time decay for the space semi-discretization of a locally damped wave equation via an artificial numerical viscosity. Numer. Math., 95(3):563-598, 2003.

[36] Louis T. Tebou and Enrique Zuazua. Uniform boundary stabilization of the finite difference space discretization of the $1-d$ wave equation. Adv. Comput. Math., 26(1-3):337-365, 2007.

[37] Marius Tucsnak and George Weiss. Observation and control for operator semigroups. Birkhäuser Advanced Texts: Basler Lehrbücher. [Birkhäuser Advanced Texts: Basel Textbooks]. Birkhäuser Verlag, Basel, 2009.

[38] Enrique Zuazua. Propagation, observation, and control of waves approximated by finite difference methods. SIAM Rev., 47(2):197-243 (electronic), 2005. 